\title{
The Electric Field and Waves Instruments on the Radiation Belt Storm Probes Mission
}

\author{
J.R. Wygant · J.W. Bonnell · K. Goetz • R.E. Ergun · F.S. Mozer · S.D. Bale • \\ M. Ludlam • P. Turin · P.R. Harvey • R. Hochmann · K. Harps • G. Dalton • \\ J. McCauley · W. Rachelson • D. Gordon - B. Donakowski - C. Shultz • C. Smith • \\ M. Diaz-Aguado - J. Fischer · S. Heavner · P. Berg • D.M. Malsapina - M.K. Bolton • \\ M. Hudson - R.J. Strangeway · D.N. Baker · X. Li · J. Albert · J.C. Foster • \\ C.C. Chaston - I. Mann - E. Donovan - C.M. Cully • C.A. Cattell • V. Krasnoselskikh • \\ K. Kersten - A. Brenneman · J.B. Tao
}

Received: 21 February 2013 / Accepted: 22 July 2013 / Published online: 11 October 2013

(C) The Author(s) 2013. This article is published with open access at Springerlink.com

J.R. Wygant $(\bowtie) \cdot$ K. Goetz · C.A. Cattell · K. Kersten · A. Brenneman School of Physics and Astronomy, University of Minnesota, Minneapolis, MN, USA e-mail: wygan001@umn.edu

J.W. Bonnell · F.S. Mozer · S.D. Bale · M. Ludlam · P. Turin · P.R. Harvey · R. Hochmann · K. Harps · G. Dalton · J. McCauley · W. Rachelson · D. Gordon · B. Donakowski · C. Shultz · C. Smith · M. Diaz-Aguado $\cdot$ J. Fischer $\cdot$ S. Heavner $\cdot$ P. Berg $\cdot$ C.C. Chaston · J.B. Tao Space Sciences Laboratory, University of California, Berkeley, CA, USA

F.S. Mozer · S.D. Bale

Department of Physics, University of California, Berkeley, CA, USA

R.E. Ergun · D.M. Malsapina · M.K. Bolton · D.N. Baker · X. Li

Laboratory for Atmospheric and Space Physics, University of Colorado, Boulder, CO, USA

R.J. Strangeway

IGPP and Department of Earth and Space Sciences, University of California, Los Angeles, CA, USA

M. Hudson

Department of Physics and Astronomy, Dartmouth College, Hanover, NH, USA

J. Albert

AFGL/RVBX, Kirkland Air Force Base, NM, USA

I. Mann

Department of Physics, University of Alberta, Edmonton, Alberta, Canada

E. Donovan · C.M. Cully

Department of Physics and Astronomy, University of Calgary, Alberta, Canada

J.C. Foster

Haystack Observatory, MIT, Cambridge, MA, USA

V. Krasnoselskikh

LPC2E, Orleans, France 
Abstract The Electric Fields and Waves (EFW) Instruments on the two Radiation Belt Storm Probe (RBSP) spacecraft (recently renamed the Van Allen Probes) are designed to measure three dimensional quasi-static and low frequency electric fields and waves associated with the major mechanisms responsible for the acceleration of energetic charged particles in the inner magnetosphere of the Earth. For this measurement, the instrument uses two pairs of spherical double probe sensors at the ends of orthogonal centripetally deployed booms in the spin plane with tip-to-tip separations of 100 meters. The third component of the electric field is measured by two spherical sensors separated by $\sim 15 \mathrm{~m}$, deployed at the ends of two stacer booms oppositely directed along the spin axis of the spacecraft. The instrument provides a continuous stream of measurements over the entire orbit of the low frequency electric field vector at 32 samples/s in a survey mode. This survey mode also includes measurements of spacecraft potential to provide information on thermal electron plasma variations and structure. Survey mode spectral information allows the continuous evaluation of the peak value and spectral power in electric, magnetic and density fluctuations from several $\mathrm{Hz}$ to $6.5 \mathrm{kHz}$. On-board cross-spectral data allows the calculation of field-aligned wave Poynting flux along the magnetic field. For higher frequency waveform information, two different programmable burst memories are used with nominal sampling rates of 512 samples/s and $16 \mathrm{k}$ samples/s. The EFW burst modes provide targeted measurements over brief time intervals of 3-d electric fields, 3-d wave magnetic fields (from the EMFISIS magnetic search coil sensors), and spacecraft potential. In the burst modes all six sensor-spacecraft potential measurements are telemetered enabling interferometric timing of small-scale plasma structures. In the first burst mode, the instrument stores all or a substantial fraction of the high frequency measurements in a 32 gigabyte burst memory. The sub-intervals to be downloaded are uplinked by ground command after inspection of instrument survey data and other information available on the ground. The second burst mode involves autonomous storing and playback of data controlled by flight software algorithms, which assess the "highest quality" events on the basis of instrument measurements and information from other instruments available on orbit. The EFW instrument provides 3-d wave electric field signals with a frequency response up to $400 \mathrm{kHz}$ to the EMFISIS instrument for analysis and telemetry (Kletzing et al. Space Sci. Rev. 2013).

Keywords Electric fields · Magnetosphere

\section{Introduction}

The goal of the Electric Field and Waves (EFW) Investigation on the Radiation Belt Storm Probe (RBSP) mission is to understand the role of electric fields in driving energetic particle acceleration, transport, and loss in the inner magnetosphere of the Earth. The overall EFW instrument development was led by the University of Minnesota under Principal Investigator, John Wygant, and Project Manager, Keith Goetz. The hardware and software development of the RBSP EFW instrument including the Instrument Data Processing Unit (IDPU) and the spin plane and spin axis boom deployment units was carried out by a team led by John Bonnell at the Space Sciences Laboratory at the University of California, Berkeley. A team at the Laboratory for Atmospheric and Space Physics at the University of Colorado led by Robert Ergun provided a signal processing board for digital filtering and on-board calculation of spectral products.

The EFW instrument provides electric field measurements on each of the two RBSP spacecraft focusing on the frequency range from DC to $256 \mathrm{~Hz}$. In addition, the instrument 
has the capability to sample large amplitude $3 \mathrm{~d}$ electric fields and magnetic fields waves and structures at $16.4 \mathrm{k}$ samples/s in special high rate mode. The frequency range of primary focus from dc to $256 \mathrm{~Hz}$ includes measurements of the convection electric field, interactions with interplanetary shocks, electric fields associated with particle injection fronts, Alfven waves, magnetosonic waves, electromagnetic ion cyclotron (EMIC) waves, lower hybrid waves. The candidate structures and waves that have been proposed for the acceleration of different particle populations in the inner magnetosphere span over seven orders of magnitude in time and four orders of magnitude in amplitude. The generation of these waves and their ability to accelerate different particle populations is often tied to the dynamics of the inner magnetosphere during geomagnetic storms. During these times, the free energy sources available to drive magnetospheric processes increase by $\sim 2$ orders of magnitude (Axford 1976) due to encounters powerful interplanetary shocks, coronal mass ejections, and fast-slow streams in the solar wind. During major geomagnetic storms, the plasma pressure between $2 R_{\mathrm{E}}$ to $6 R_{\mathrm{E}}$ is dominated by the development of a hot (20-700 keV) ion ring current torus surrounding the Earth which is generated by intense convection electric fields that appear deep within the magnetosphere. The spatial configuration of the convection electric fields is modified by the development of powerful ring current pressure gradients in a complex interplay of self-consistent dynamics that ultimately controls the cold plasma density structure, the intensity and configuration of the magnetic field, and the ability of the system to support a wide variety of wave modes. A signature feature of major geomagnetic storms is the erosion of the plasmasphere on the night-side to within $2 R_{\mathrm{E}}$ of the Earth in the equatorial plane. During this massive reconfiguration of the inner magnetosphere, relativistic particle acceleration and loss mechanisms are particularly strong and variable. More than half a dozen different acceleration mechanisms with varying degrees of theoretical and experimental support have been invoked to explain the several order of magnitude increases and decreases in relativistic and near relativistic electrons and energetic ions.

In this section, we present examples of electric field measurements and their limitations from the CRRES spacecraft to illustrate the varied roles of the electric field in the inner magnetosphere. These data motivated the measurement goals of the RBSP EFW instruments. Next we present recently obtained on-orbit data from the first few months of the RBSP mission that illustrate instrument capabilities. In subsequent sections, we present the measurement goals of the instrument, followed by an instrument overview and a more detailed discussion of the instrument subsystems.

Figure 1 presents a pass from the CRRES spacecraft through the inner magnetosphere in the equatorial plane during the major geomagnetic storm of March 1991. These data illustrate three different energization processes that produced dramatic effects on different particle populations. The three processes are: (1) interplanetary shock induced acceleration; (2) anomalously large enhancements in the convection electric field; and (3) large amplitude ULF waves present throughout the inner magnetosphere. This figure shows an abrupt enhancement in the electric field of $40 \mathrm{mV} / \mathrm{m}$ on the night-side of the Earth near $L=2.4$ with a duration of 60 seconds associated with an interplanetary shock induced magnetosonic wave (Wygant et al. 1994). It is estimated that the amplitude of the magnetosonic wave on the dayside, where it was directly incident on the inner magnetosphere, was on the order of $250 \mathrm{mV} / \mathrm{m}$. Energetic electron measurements on CRRES showed (Blake et al. 1992; Blake and Imamoto 1992; Vampola and Korth 1992) that this structure produced a five order of magnitude enhancement in $>13 \mathrm{MeV}$ electrons over a period of $<1$ minute that evolved into a new radiation belt near $2 R_{\mathrm{E}}$ that lasted over 2 years. The shock induced prompt acceleration of the relativistic electrons was subsequently reproduced in detail by large-scale test particle simulations (Li et al. 1993; Hudson et al. 1997). Shock induced acceleration of protons was also observed during this event and simulated (Hudson et al. 1995). 
The shock induced magnetosonic wave created a 5 order of magnitude increase in 13 $\mathrm{MeV}$ electron fluxes in $<100$ seconds resulting in a new radiation belt that lasted two years

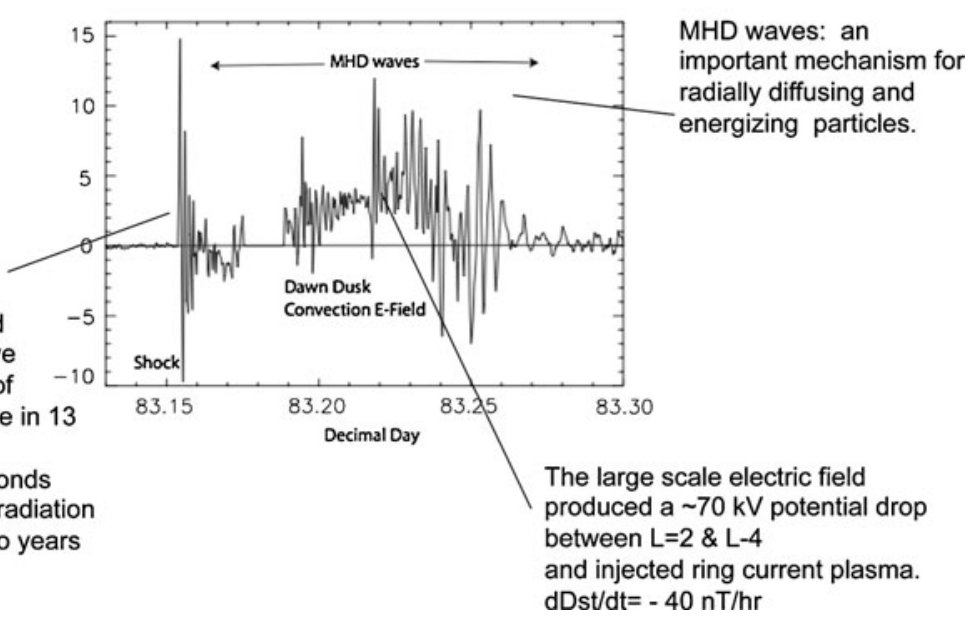

Fig. 1 CRRES measurements during major geomagnetic storm of March 21, 1991 showing interplanetary shock induced electric fields, the enhancement in the large scale electric field, and ULF waves. Inset figure shows the CRRES orbit with "explosion symbol" indicating position of spacecraft at time of encounter with shock induced magnetosonic wave and tip of red arrow indicating end of plot interval. Text indicates the kinds of energization expected from each structure

Figure 1 also shows an enhancement in the large-scale dawn-dusk convection electric field from quiet time values of $0.1 \mathrm{mV} / \mathrm{m}$ to values of $5-7 \mathrm{mV} / \mathrm{m}$ during the main phase of the storm. Measurements of the magnetic field perturbations associated with ring current plasma provide evidence that the plasma was injected by this electric field to a radial distance of $\sim 2 R_{\mathrm{E}}$ over a several hour period (Wygant et al. 1998; Rowland and Wygant 1998). Radar measurements at ionospheric altitudes also provide evidence for similar storm time enhancements of the large-scale convection electric field at low latitudes (Yeh et al. 1991).

Finally, Fig. 1 shows strong MHD fluctuations of $\sim 10 \mathrm{mV} / \mathrm{m}$ lasting almost the entire orbit that in principle could interact strongly with $\sim 1 \mathrm{MeV}$ electrons stochastically to produce radial diffusion and energization through conservation of the first adiabatic invariant. Alternatively, the acceleration could proceed through a "coherent" non-linear interaction involving trapping in the quasi-monochromatic large amplitude waves. In order to understand the role of ULF waves in the energization of radiation belt particles, the amplitude of the electric field, its propagation velocity, its azimuthal wave number, and its radial spatial scales must be measured (Hudson et al. 2001, 2004; Brautigam et al. 2005). These properties should be measured as a function of radial position and local time. In addition, the mechanisms responsible for the generation of the waves should be understood. Candidate mechanisms for generating these waves include magnetopause surface perturbations driven by solar wind dynamic pressure variations, reconnection at the front-side magnetopause, large scale shear mode instabilities on the flanks of the magnetopause, the internal free energy in anisotropic ring current ion distribution functions, and stochastic forcing by substorm related electric fields. The existence of the two RBSP spacecraft, upstream solar wind monitors, and the routine presence of the THEMIS spacecraft at larger radial distances will be used to evaluate the driving forces, the structure of the waves, and to distinguish between competing accelerating mechanisms. 
Evidence from CRRES and other spacecraft also has shown that substorm injection fronts propagating inward from the tail can also produce electric fields ranging from $1-40 \mathrm{mV} / \mathrm{m}$ for periods of 1 to 5 minutes (Rowland 2002; Dai et al. 2011; Li et al. 1998). These fronts could result in the injection and energization of both ring current ions and high-energy electrons (10-700 keV), which are the dominant contribution to the particle energy density and pressure around the Earth during major geomagnetic storms. There is evidence (Ingraham et al. 2001) suggesting that intense substorm injection fronts may be able to energize significant fluxes of electrons up to $>1 \mathrm{MeV}$ below $L=4-5$ over time scales of minutes. However, our understanding of the efficiency of the mechanism is largely based on CRRES, which only saw one very large storm on the night side of the Earth. In addition, because CRRES was a single spacecraft, it could not measure the propagation velocity of the injection event or the radial and azimuthal extent of the region of accelerating electric fields.

Figure 2 presents measurements of the dawn-dusk electric field from the EFW instrument on RBSP-A and of the energetic electron omni-directional fluxes from the MagEIS instrument during an injection event associated with a small storm on January 17, 2013. The fluctuating electric field is significantly enhanced over the four hour time interval from 18:00 UT to 22:00 UT, often exceeding $10 \mathrm{mV} / \mathrm{m}$. There is also am average positive value of the Ey mgse (or dawn-dusk) electric field component of 1-3 mV/m beginning at 14 UT and lasting about two hours until the spacecraft move earthward to lower l-values. The dc field is seen again on the out bound pass from 18 UT to the end of the day. Thus the electric field is observed nearly continuously as the spacecraft moves from midnight to the morning sector. The total period of enhanced dawn-dusk electric fields is nearly eight hours. This period coincides with a decrease in Dst from 33 at 14:00 UT to -53 at 24:00 indicating an injection of ring current plasma during this period of strong electric fields. While there have been a number of studies of large amplitude convection and ULF electric fields in the tail and on the night side near geosynchronous orbit, to our knowledge, these are the first observations showing that such large fields can be nearly continuously present for many hours. A cursory examination of this and similar time intervals provides evidence for a rich phenomenology of magnetic field dipolarizations, plasma-sheet expansions and contractions, encounters with the plasma sheet boundary layers, Alfven waves and other ULF waves, and enhancements in the large scale convection electric field. The second panel of Fig. 2 shows line plots of flux from selected MagEIS electron energy channels ranging between $37 \mathrm{keV}$ and $846 \mathrm{keV}$. The third panel presents color-coded electron fluxes from the MagEIS instrument (Blake et al. this issue) in an energy time spectrogram format. The data from the second and third panel suggest an abrupt injection of $<100 \mathrm{keV}$ electrons during the period of the enhanced quasi-static and fluctuating electric field. At the same time, the particles fluxes with energies larger than $100 \mathrm{keV}$ are decreased by about an order of magnitude. This behavior illustrates the complexity of response of different energy particles to electric fields that vary over different spatial and temporal scales. Much detailed analysis remains to unravel the complicated phenomenology revealed by this data set.

The lower values of the electric field, with magnitude less than $1 \mathrm{mV} / \mathrm{m}$ seen over the interval from 9:00 to 12:00 UT in Fig. 2, indicate the sensitivity of the electric field measurement is a fraction of a $\mathrm{mV} / \mathrm{m}$. Evaluations of measurements from orbits occurring during especially quiet periods $(K p<1)$ indicate the spin-plane electric measurement is sensitive to fields of $<0.3 \mathrm{mV} / \mathrm{m}$.

The CRRES spacecraft had only limited capability of making high time resolution burst measurements, so that intense small-scale wave fields that might violate the conservation of the first or second adiabatic invariants within the injection front could not be evaluated. The RBSP EFW electric field measurements along with measurements of the magnetic field 

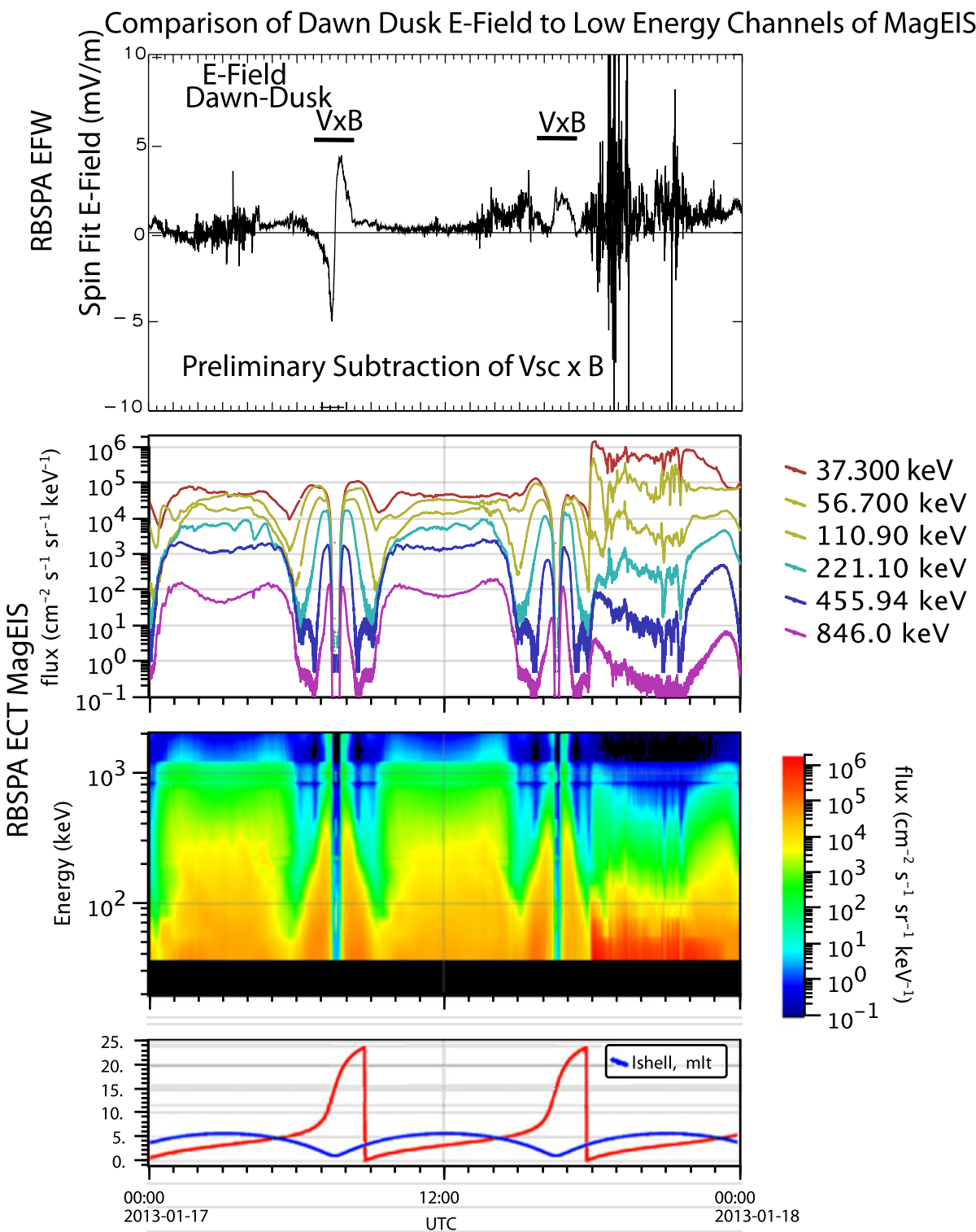

Fig. 2 RBSP-A measurements from January 17, 2013 of the (upper panel) dawn-dusk component of the electric field from the spin plane booms at a spin period cadence $(11.5 \mathrm{~s})$. The second panel presents the omni-directional electron energy flux from selected energy channels ( $37 \mathrm{keV}$ to $846 \mathrm{keV}$ ) from the MagEIS instrument. The third panel presents a color-coded omni-directional fluxes in a energy-time spectrogram over the lower energy channels of the MagEIS instrument (MagEIS data courtesy J. Fennell, H. Spence and J.B. Blake). The bottom panel consists of line plots of the spacecraft L-value and Magnetic Local Time (MLT) in units of hours

and energetic particles from other instruments will determine, for the first time, the global spatial structure and time evolution of injection events as they propagate through the inner 
magnetosphere. This information is crucial for assessing the ability of diverse structures to accelerate particles of different energies and inject them deep into the inner magnetosphere.

At higher frequencies, the EFW instrument will measure electric fields associated with kinetic Alfven waves, lower hybrid waves, ion cyclotron waves, and small-scale discrete structures such as solitary waves. Since CRRES, a number of studies have suggested that whistler mode chorus with amplitudes of 0.1 to several $\mathrm{mV} / \mathrm{m}$ can be effective in the stochastic energization of electrons over $100 \mathrm{keV}$ to $>1 \mathrm{MeV}$ (Roth et al. 1999; Horne and Thorne 1998; Albert 2000; Summers and Omura 2007). It has only been in recent years that the possible role of large amplitude small-scale or high frequency waves in the prompt energization and loss of charged particles over broad energy ranges has been appreciated. High time resolution electron measurements from SAMPEX (Blake et al. 1996; Lorentzen et al. 2002) have provided evidence for relativistic microbursts, which consist of abrupt enhancements of relativistic electron precipitation over periods small compared to an electron bounce period. Measurement of bremsstrahlung x-rays due to precipitating high energy electrons have been provided by balloon-borne detectors and have similarly shown that relativistic electrons can be scattered into the loss cone on rapid time scales (Millan et al. 2002).

Figure 3 from Cattell et al. (2008) presents recent STEREO high time resolution burst measurements of very large amplitude $(200 \mathrm{mV} / \mathrm{m})$ obliquely propagating whistler mode waves in the inner magnetosphere just outside the plasmapause. Panel A presents a colorcoded frequency time spectrogram showing the over all interval of whistler mode power. The lower plot of panel $\mathrm{A}$ is the output of a peak detector that provides the peak amplitude each minute of the whistler mode waves. These measurements indicate large amplitude waves at a frequency of $\sim 0.2 f_{\text {ce }}$ (the electron cyclotron frequency, plotted in black) are present for about 30 minutes. Panel B provides a 0.5 second high time resolution measurement of an intense whistler mode pulse from the S/WAVES time domain sampler at a resolution of $35 \mathrm{k}$ samples/s. Panel D provides evidence from SAMPEX of microburst activity on "nearly" conjugate field lines (within 1 hour MLT and 10 minutes) as the STEREO observations. Panel C provides results from a 1-D test particle simulation in which a large amplitude whistler wave is launched along a magnetic field line and interacts with a $1 \mathrm{MeV}$ electron with an initial pitch angle of $30^{\circ}$ through a cyclotron resonant interaction. The top panel plots kinetic energy in $\mathrm{MeV}$, the second panel plots the resonance mismatch (see Roth et al. 1999), the third panel plots the equatorial pitch angle, the bottom panel plots the distance along the field line from the equatorial plane in $\mathrm{km}$ and the $\mathrm{x}$-axis is time from 0 to 2 seconds, with the electron initially at the equatorial plane. The figure shows that the whistler traps the electron and accelerates it up to $4 \mathrm{MeV}$ in about 0.2 seconds (first panel). The wave also causes strong pitch angle scattering (third panel). The results from these simple test particle simulations indicate that large amplitude whistler mode waves may be capable of nonlinear trapping and acceleration of electrons from initial energies of $100 \mathrm{keV}$ to final energies of $2 \mathrm{MeV}$ over broad portions of phase space in fractions of a second (Cattell et al. 2008) in a single interaction. Over 20 one-half second intervals of large amplitude whistler waves were observed by the STEREO spacecraft during one pass associated with a modest substorm. More evidence for intense whistlers was found by Cully et al. (2008) using the THEMIS spacecraft and, by Kellogg et al. (2010) and Wilson et al. (2011) utilizing Wind data. Evidence for an association of the large amplitude whistler waves with microbursts has been provided by Kersten et al. (2011) using roughly simultaneous measurements from the STEREO spacecraft and the low altitude SAMPEX spacecraft and also measurements between WIND and SAMPEX. An understanding of how the waves are generated and their ability to accelerate particles has been limited by the lack of simultaneous high cadence 


\section{Whistler Mode Wave Observations from STEREO}
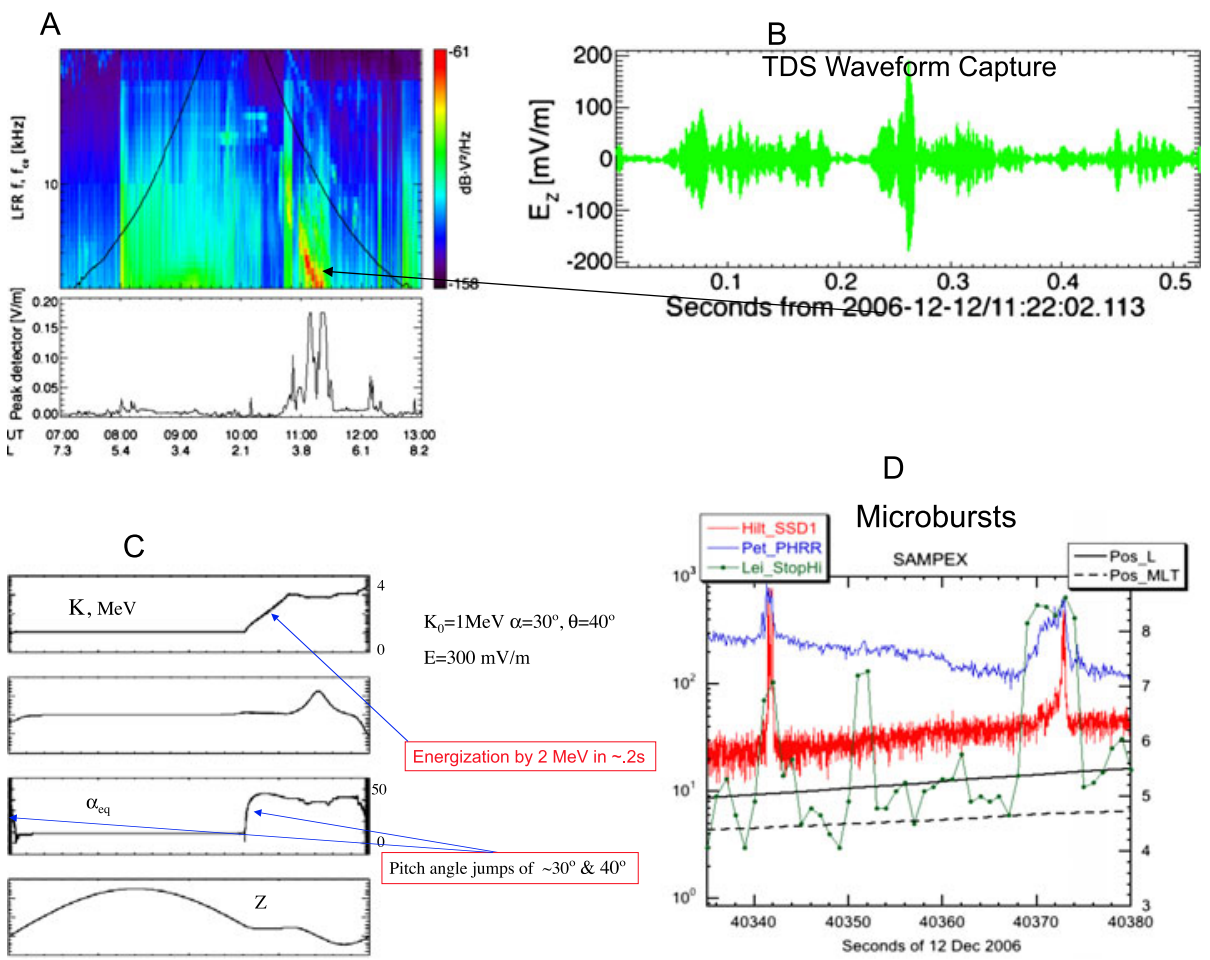

Fig. 3 Wave measurements from STEREO motivating measurements goals of RBPS-EFW. Panel A: Upper plot is the electric field spectrogram over frequency range from $2 \mathrm{kHz}$ to $100 \mathrm{kHz}$ (Cattell et al. 2008) from S/WAVES STEREO spacecraft during pass through inner magnetosphere showing strongly enhanced power in whistler band near $L=3-4$ at about $11 \mathrm{UT}$; Panel $\mathbf{A}$, lower plot is the peak amplitude in electric field associated with enhanced whistler waves measured once per minute by the peak detector. Panel B: High time resolution waveform capture from STEREO showing large $(200 \mathrm{mV} / \mathrm{m}$ ptp) whistler wave packets from burst recording at time indicated on spectrogram. Panel $\mathbf{C}$ : Test particle simulations of imposed whistler wave field of amplitude $300 \mathrm{mV} / \mathrm{m}$ interacting with an electron with initial energy $1 \mathrm{MeV}$. The top plots is the kinetic energy in $\mathrm{MeV}$, the second plot is the resonance mismatch (see Roth et al. 1999), the third plot is the equatorial pitch angle, the bottom plot is the distance along the field line from the equatorial plane in $\mathrm{km}$ and the $\mathrm{x}$-axis is time from 0 to 2 seconds. The top plot shows the abrupt coherent nonlinear energization of electrons by $2 \mathrm{MeV}$ and the third plot shows the jump in pitch angle through cyclotron resonance during one interaction. Panel D: Near simultaneous (within 10 minutes) low altitude SAMPEX observations of relativistic electron microbursts over same L value extent as STEREO whistler displaced 1 hour in magnetic local time

energetic particle measurements and field measurements on the same spacecraft. The EFW instrument provides unambiguous measurements of the occurrence frequency of large amplitude waves, as well as their spatial distribution along magnetic field lines, in radial distance, and in local time. The high time resolution particle measurements will be provided on RBSP by the MAGEIS instrument (Blake et al. this issue) and HOPE instrument (Reeves et al. this issue).

The EFW instrument has the capability of measuring, through burst recordings, large amplitude (requirement $<500 \mathrm{mV} / \mathrm{m}$; capability $>4 \mathrm{~V} / \mathrm{m}$ ) waves and structures at frequencies up to $16.4 \mathrm{k}$ samples/s and recording them in burst memory. These will be compared to high time resolution measurements of particles by the HOPE and MagEIS instruments on RBSP 
and also to measurements of microburst X-rays detected by balloon-borne instrumentation flown during the BARREL campaign (Millan this issue).

Early measurements from the EFW instruments on RBSP during several major storms illustrate that large amplitude whistler waves routinely have peak values ranging from 10 to $>200 \mathrm{mV} / \mathrm{m}$ over the entire orbital path outside the plasmasphere during active periods. The incidence of high amplitude whistler waves systematically enhances during major storm periods from apogee at $5.8 R e$ to radial distances below $3 R e$, spanning the position of the outer radiation belts. Figure 4 presents measurements from the main phase of a geomagnetic storm on November 1, 2012. The top panel shows line plots of flux from selected MagEIS electron energy channels ranging between $37 \mathrm{keV}$ and $1942 \mathrm{keV}$ (Spence et al. this issue; Blake et al. this issue); the second panel presents the omidirectional electron fluxes from the MagEIS in an energy time spectrogram format showing a series of injection events (labeled "INJECT"), the outer radiation belts ("OB") and the slot region between the outer and inner belts. Notice that during the final orbit the energetic electrons display a complex signature. Near apogee, between 18:00-20:00 UT, the lower energy (36 and $57 \mathrm{keV}$ ) electron fluxes near apogee are strongly enhanced over the previous orbit. The higher energy electrons (100 $\mathrm{keV}$ to $2 \mathrm{MeV}$ ) fluxes decrease relative to those of the previous orbit. This complex behavior is the consequence of the interplay between a variety of different acceleration and loss mechanisms, including enhancements in the large-scale convection electric field, changes in the magnetic field configuration, current sheet scattering, magnetopause shadowing, and higher frequency wave activity. Here we emphasize observations of the waves. The next two panels present frequency-time spectrograms of the spectral power in the wave electric and magnetic fields (respectively). The wave magnetic field is measured by the magnetic search coil sensor in the EMFISIS instrument (Kletzing et al. 2013) and provided to the $\mathrm{EFW}$ instrument via an analog interface. The electric and magnetic spectrograms show that there are enhanced whistler mode waves (labeled "whis"), which extend from near apogee down to radial distances coinciding with the outer radiation belts. Whistler mode chorus is thought to be generated at the magnetic equator and is usually observed in two bands, an upper band and a lower band centered on $f_{\text {ce }} / 2$ at the equator. The second orbit during this day is near the magnetic equator and provides, as expected, the upper and lower band separated by the measured $f_{\mathrm{ce}} / 2$. However, during the last orbit, which occurs during the main phase of the storm, the spacecraft is at higher magnetic latitude and the instrument observes only the lower band, and its upper limit frequency is much less than the locally measured $f_{\text {ce }} / 2$. This suggests the possibility, subject to further analysis and observations from other time intervals, that the value of the magnetic field at the equator where the chorus is generated is much less than the locally measured magnetic field and thus the frequency of the waves, which (to a good approximation) have group velocities along magnetic field lines and propagate to the spacecraft along the field, reflects the equatorial $f_{\mathrm{ce}} / 2$. These deviations in the frequency of the whistlers occur almost exclusively during storms when field lines are likely to be especially stretched. They may provide information on the accuracy of magnetic field models and on the adiabatic deceleration of energetic particles, which respond to field line stretching. Such stretching might also enhance magnetopause shadowing and current sheet scattering loss mechanisms. Another possibility, that should be investigated with ray tracing programs, is that the whistler mode waves are not propagating along magnetic field lines, in this especially distorted magnetic field configuration. All these issues need further careful study and are beyond the scope of this initial paper.

Electromagnetic emissions seen in both the electric field and the search coil between the hydrogen gyro-frequency $(\sim 100 \mathrm{~Hz})$ and the lower hybrid frequency $(\sim 1 \mathrm{kHz})$ are enhanced at 14:00 UT during an inbound approach to perigee and again at 23:00 UT during 


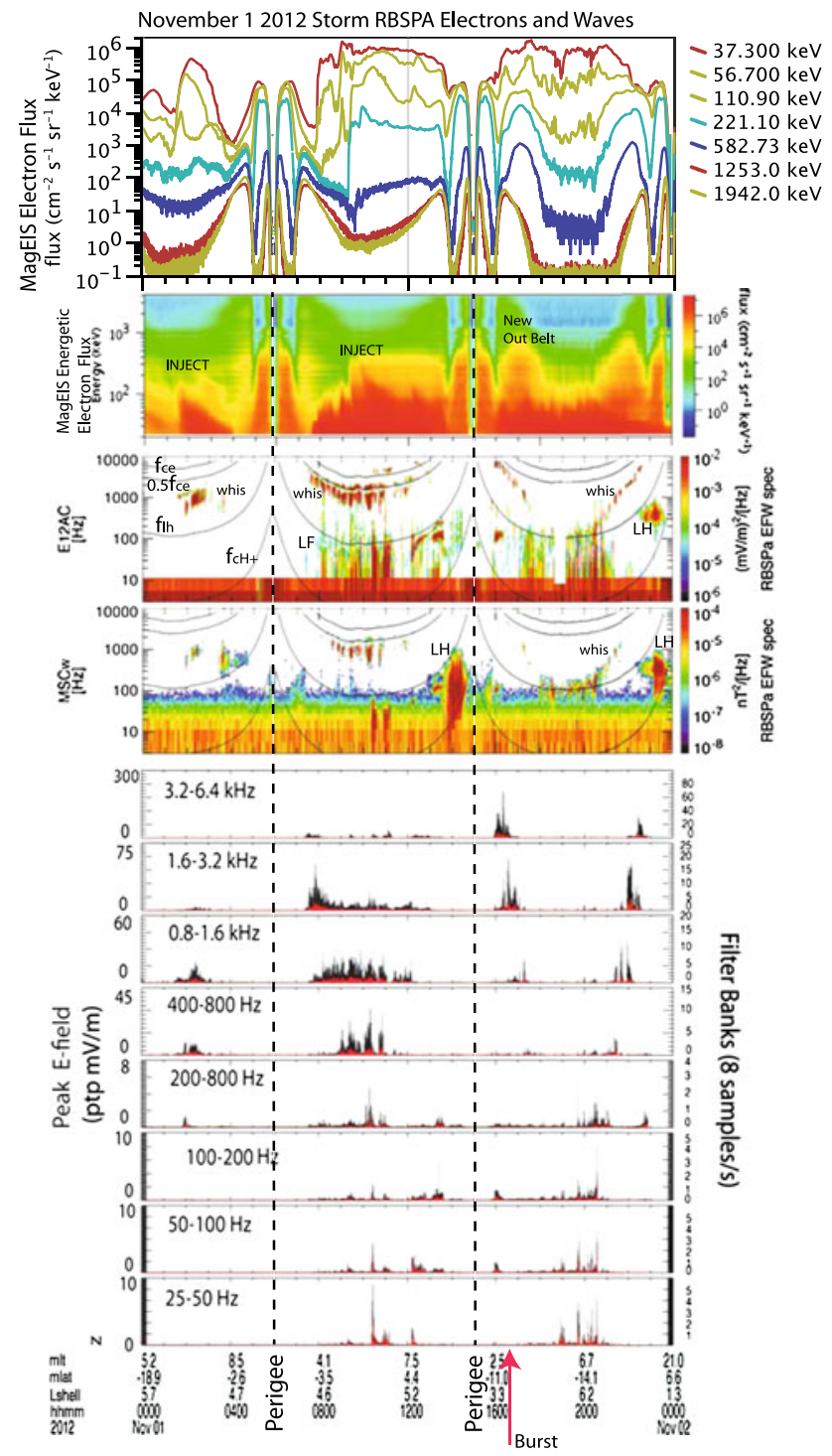

Fig. 4 Measurements during the geomagnetic storm on 11/01/2012 from RBSP-A. Top panel is energetic electron omni-directional flux from selected energy channels from the MagEIS instrument and the second panel is the color-coded omni-directional fluxes in a energy-time spectrogram format from $37 \mathrm{keV}$ to $4 \mathrm{MeV}$. The third and fourth panels are frequency-time spectrograms showing (third panel) the power in the wave electric field from a pair of E-field booms and (fourth panel) the power in the wave magnetic field from a single search coil magnetometer sensor from the EMFISIS instrument as telemetered through EFW. The subsequent eight panels contain line plots of the peak-to-peak value of the electric field at 8 samples/s in each of a series of frequency bins ranging from $25 \mathrm{~Hz}$ to $6.4 \mathrm{kHz}$ in order of decreasing frequency. The vertical scale is variable for each bin and includes the calibrations of instrument frequency response and effective antenna length. The vertical red line coincides with the time of the burst data in the next figure. The wave data from the storm period on 11/01/2012 showing intense average wave power and extra-ordinarily large values of the peak whistler amplitudes over the radial distance from $3 \operatorname{Re}$ to $5.8 \operatorname{Re}$ (apogee). The dark curves superimposed on the wave spectrograms correspond to (in sequence from lowest to highest frequency) the hydrogen ion gyro-frequency, the lower hybrid frequency, one half of the electron gyro-frequency, and the electron gyro-frequency 
another inbound approach. These strongly enhanced waves coincide with the position of the "New Outer Belt" which appeared at 14:00 UT. Spacecraft potential measurements (not shown) indicate that these waves are observed inside the plasmasphere. It is worth noting that magnetosonic waves and waves near the lower hybrid frequency inside the plasmasphere are candidate waves for the stochastic acceleration of electrons between $100 \mathrm{keV}$ and several MeV (Horne and Thorne 1998).

Strong electric and magnetic fields are also present at apogee between the ion cyclotron and lower hybrid frequencies. These lower frequency waves (labeled "SS" for "Small Scale") are in the frequency range of lower hybrid waves, ion cyclotron waves, kinetic Alfven waves, and magnetosonic waves. The bottom set of 8 panels in Fig. 4 show the peak electric field measurements in selected frequency bins ranging from $20 \mathrm{~Hz}$ to $6.4 \mathrm{kHz}$. These electric field peak detectors are sampled routinely with unprecedented time resolution; each channel is sampled 8 samples/s compared to 1-2 samples per second or once per spin on previous spacecraft electric field instruments. The RBSP sampling is comparable to the time interval between individual wave pulses and allows us to count high amplitude whistlermode and other wave mode pulses. These peak measurements will enable assessment of the role of large amplitude whistler waves in the non-linear scattering and energization of energetic electrons and provide context for burst measurements of electric fields (see, for example, burst at time of red arrow shown in Fig. 5). In this plot, they reveal that intense whistler waves with amplitudes exceeding $100 \mathrm{mV} / \mathrm{m}$ (peak-to-peak) are present before and during periods of enhanced outer belt fluxes and extend over the entire spatial extent of the outer zone radiation belts down to the slot region. These are necessary conditions for the waves to be important in accelerating outer zone energetic electrons.

Routine high-time resolution burst measurements of electric field waveforms from RBSP allow a detailed examination of wave properties. Figure 5 shows 0.8 seconds of data (from a total burst duration of 5 seconds sampled at $16.4 \mathrm{k}$ samples/second) obtained on the November 1, 2012 storm period at 16:20:26 UT (red arrow in Fig. 4) from the RBSP-A EFW burst memory. The figure shows packets of large amplitude whistler wave with peak to peak amplitudes exceeding $200 \mathrm{mV} / \mathrm{m}$.

As previously discussed, the structure and dynamics of the plasmasphere are important for understanding the dynamics of the inner magnetosphere during major geomagnetic storms. The plasmasphere can be strongly eroded during major geomagnetic storms by the action of the large-scale convection electric field. During these times, the plasmapause can move from its quiet time position of $>5 R e$ to within $2 R e$. However, there have been few direct experimental comparisons in the outer magnetosphere between the large-scale electric field and the plasmaspheric structure. Information on cold plasma densities is provided on the RBSP mission by tracking the frequency of the upper hybrid line as determined by the EMFISIS instrument and by the measurement of the spacecraft potential by EFW. The EMFISIS determination of density is more accurate than the density estimated from spacecraft potential and less subject to uncertainties associated with cold electron temperature effects. The spacecraft potential measurement provides higher time resolution ranging up to 16 samples/s in routine survey mode. Figure 6 presents a cross calibration over one day and several orbits between density determined the upper hybrid line and that estimated from the EFW spacecraft potential. The plasmaspheric profile is clearly shown in the spacecraft potential measurements and the cold plasmaspheric density determined to better than the $50 \%$ accuracy of the measurement goal.

The two RBSP spacecraft provide measurements at radially and azimuthally separated points deep in the inner magnetosphere allowing, for the first time, the determination of the spatial extent, velocities of propagation, and temporal evolution of large scale electric field 


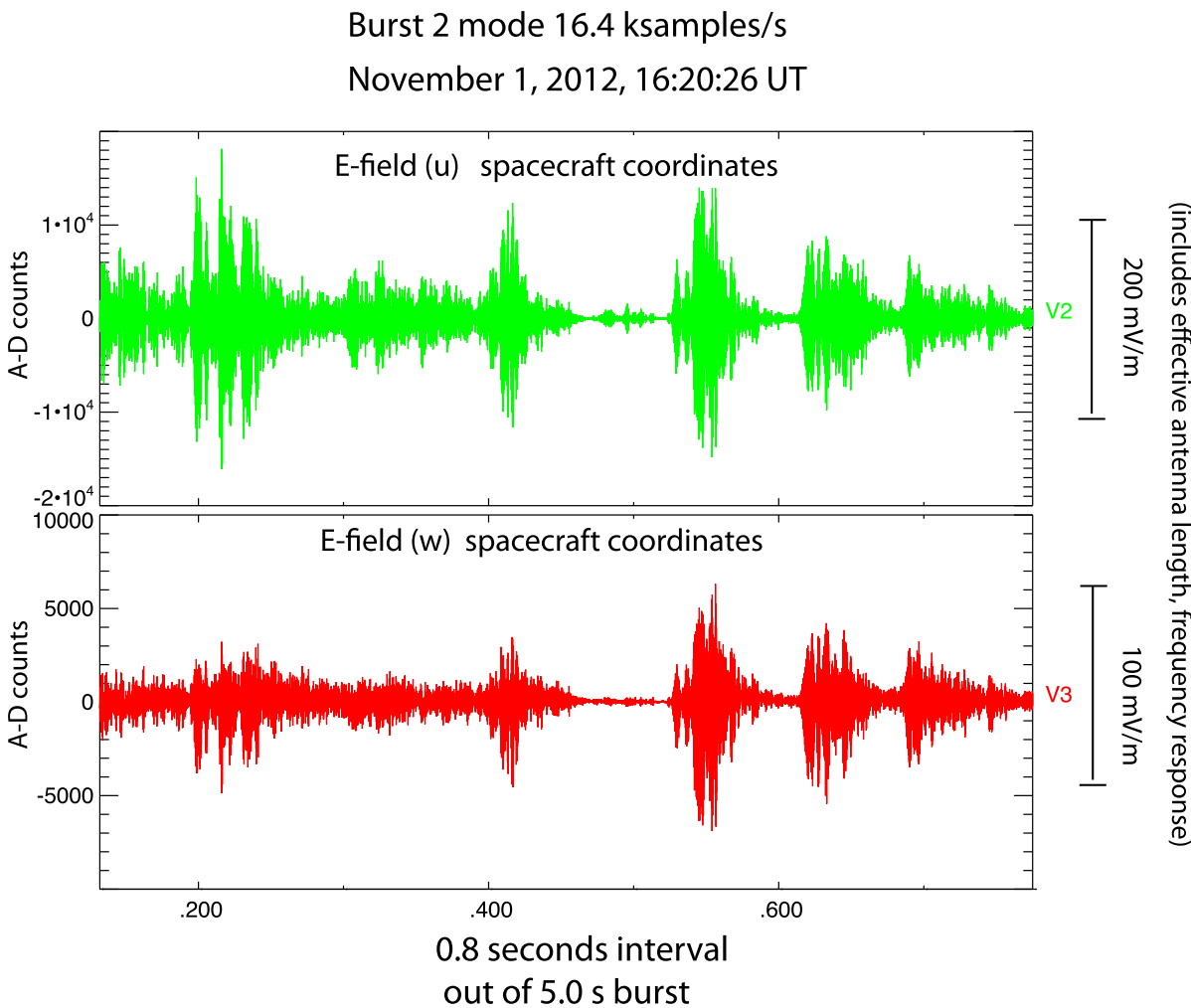

Fig. 5 EFW burst recording on 11/01/2012 16:20:26 of high time resolution measurements of two components of the electric field in the spin plane of the spacecraft showing a whistler waveform over a period of 0.8 seconds. This burst was triggered by the peak power in the electric field determined by the sum over the five highest frequency bins in the filter banks shown in the previous figure. The time of this burst coincides with the red arrow labeled "burst" at the bottom of Fig. 4. EFW bursts also include search coil magnetometer data from the EMFISIS instrument (not shown). The total burst duration was 5 seconds

structures associated with shocks, injection fronts, and MHD waves. The two spacecraft measurements will distinguish between temporal and spatial structures in the hot ring current plasma and allow the measurement of ring current pressure gradients that control the configuration of convective flows. This provides a framework for understanding the ability of different mechanisms to selectively energize different particle populations. The two spacecraft mission will determine the radial and azimuthal extent of the higher frequency wave fields thought to be important in energization and scattering. The amplitude structure, occurrence frequency, polarization, direction of propagation, E/B ratios and local density structure will be investigated in detail.

Previous magnetospheric missions missed many of these effects because the spacecraft orbits were not in the equatorial plane, they had apogees outside the region of interest, and they passed through the inner magnetosphere for only short periods of time often missing the intervals of powerful acceleration. They sometimes were not equipped with the necessary complement of electric field, magnetometer, and particle instruments. The CRRES spacecraft, which has provided some of the most interesting measurements in the inner magnetosphere, took measurements when the monitoring of upstream solar wind conditions was sporadic. There were no solar wind observations of the interplanetary shock or the CME that 
Fig. 6 Comparison of spacecraft potential to the density determined from the upper hybrid line. The top panel is the color-coded spectrogram of the wave electric field over the frequency range from about $10 \mathrm{kHz}$ to above $400 \mathrm{kHz}$ in a frequency vs time format (obtained the EMFISIS instrument). The superimposed dark line is the prediction of the upper-hybrid line based on an empirical fit to the EFW measured spacecraft potential. The bottom panel is the directly measured spacecraft potential measured as the sum of the potential measurement of the individual probes in the spin plane $(\mathrm{V} 1+\mathrm{V} 2) / 2$

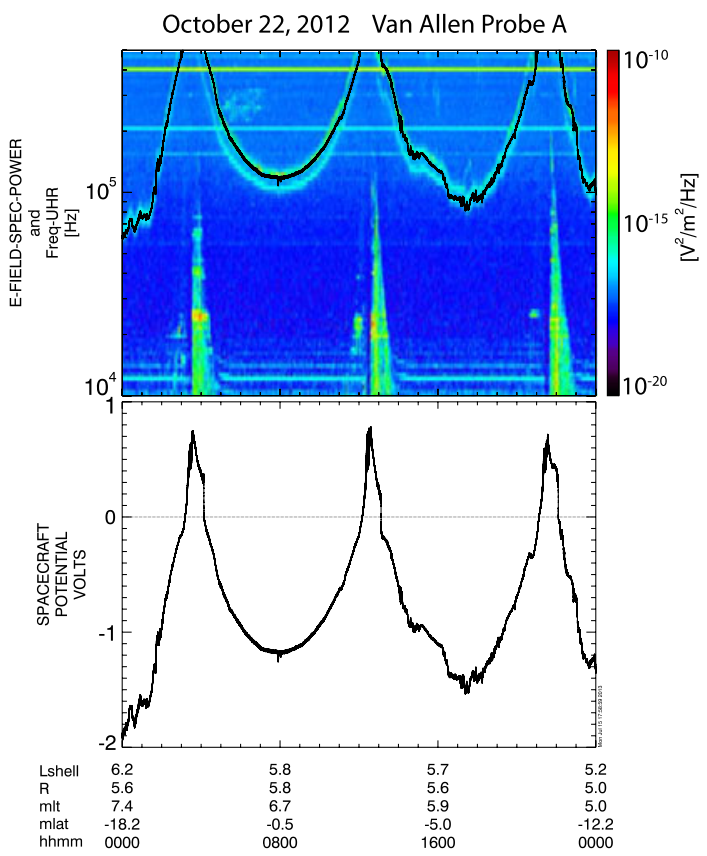

drove convective flows and ring current injection for the March 1991 storm or most of the other storms of the CRRES mission. The CRRES electric field instrument had only a small burst memory and a limited capability for high time resolution measurements of electric fields and therefore never saw the large amplitude whistler waves thought to be a major energization candidate. Some of the most important measurements of large amplitude whistler waves came from the WAVES instrument on STEREO and also the WAVES instrument on WIND. The STEREO mission was a two-spacecraft solar wind mission that only orbited through the inner magnetosphere about a half a dozen times on their way out to their respective orbits around the Sun. The observations of numerous large amplitude whistler wave packets were obtained during just one pass during a modest substorm and were not part of the prime science effort of that mission. The WIND spacecraft similarly orbited through the inner magnetosphere only a limited number of times.

Another major NASA asset that will significantly enhance the science return of EFW and other RBSP instruments is the THEMIS spacecraft, which will be able to provide crucial information on electric fields and particles at larger radial positions which provides the source population for many of the inner magnetosphere acceleration processes. The EFW team includes Co-Is associated with the electric field experiment on THEMIS. In order to enhance global coverage of the large-scale electric field and provide insight into the ionospheric effects of major geomagnetic storms, the EFW team has included the Millstone Hill radar, which provides measurements of electric field in the mid-latitude regions. In addition, the EFW team plans to coordinate high time resolution burst electric field measurements with the BARREL investigation (Millan this issue), which will provide balloon-borne measurements of X-rays associated with relativistic electron microbursts. 


\section{EFW Measurement Requirements}

In order to measure the electric fields responsible for the acceleration mechanisms described above, the EFW instrument has been designed to meet a series of demanding measurement requirements and goals. The most important requirements driving instrument design are described below. The instrument meets or exceeds these requirements.

- Measure 2-d quasi-static electric fields in the spin plane of the spacecraft at radial distances $>3 R_{\mathrm{E}}$ to an accuracy of $0.3 \mathrm{mV} / \mathrm{m}$ or $10 \%$ of the maximum electric field amplitude, which ever is larger, over a dynamic range of $\pm 500 \mathrm{mV} / \mathrm{m}$.

- To provide measurements of the quasi-static electric field component along the shorter spin axis booms to an accuracy of $4 \mathrm{mV} / \mathrm{m}$ or $20 \%$ of the maximum electric field magnitude at radial distances of $>3 R e$.

- To provide measurements of cold $(<30 \mathrm{eV})$ plasma variations in the plasmasphere over time scales from DC to $<1 \mathrm{~s}$ with an accuracy of $50 \%$ over a density range from 0.1 to $50 \mathrm{~cm}^{-3}$.

- To provide burst waveform measurements of large amplitude electric fields up to at least $250 \mathrm{~Hz}$ with an accuracy of $0.3 \mathrm{mV} / \mathrm{m}$ and a range of $500 \mathrm{mV} / \mathrm{m}$ at a cadence of $512 \mathrm{sam}-$ ples/s.

- To include measurements of the 3-d wave magnetic field obtained from the EMFISIS instrument in burst recordings (along with the wave electric field measurements described above) up to at least $250 \mathrm{~Hz}$.

- Provide interferometric timing of the propagation of small-scale waves and structures between opposing sensor pairs using burst recordings with a time cadence of up to $16.4 \mathrm{k} \mathrm{samples} / \mathrm{s}$.

- Provide spectral and cross-spectral information on the wave electric fields, magnetic fields, and density fluctuations up to $250 \mathrm{~Hz}$.

- Provide the EMFISIS wave instrument with the three measured components of the electric field over the frequency range from $10 \mathrm{~Hz}$ to $400 \mathrm{kHz}$ with a noise level of $10^{-13} \mathrm{~V}^{2} / \mathrm{m}^{2} \mathrm{~Hz}$ at $1 \mathrm{kHz}$ and $10^{-17} \mathrm{~V}^{2} / \mathrm{m}^{2} \mathrm{~Hz}$ at $100 \mathrm{kHz}$ with a $90 \mathrm{~dB}$ dynamic range and a maximum signal of $30 \mathrm{mV} / \mathrm{m}$.

\section{Overview}

\subsection{Instrument Design}

The design of the RBSP EFW instrument is based on heritage from a long line of double probe instruments including those on the S3-3 spacecraft (Mozer et al. 1979), ISEE-1 (Mozer et al. 1973), CRRES (Wygant et al. 1992), Viking (Marklund 1993), Freja (Marklund et al. 2004), FAST (Ergun et al. 2001), Polar (Harvey et al. 1995), the Cluster multispacecraft mission (Gustafsson et al. 1997), and the THEMIS spacecraft (Bonnell et al. 2008). An extensive discussion of electric field measurements and error sources of this kind of electric field instrument may be found in Bonnell et al. (2008).

On RBSP the three dimensional electric field is measured using spherical sensors at ends of two orthogonal pairs of centrifugally deployed spin plane booms with tip to tip separations of $100 \mathrm{~m}$ and a pair of spin-axis stacer booms with an adjustable tip to tip separation of 12-14 m. A schematic of the spacecraft showing the orientation of the booms is presented in Fig. 7. Also shown are the search coil and fluxgate magnetometers. Table 1 presents information on the relation between the spacecraft coordinate system and the measurement 
Spacecraft Coordinate Systems, Sensors, and Sun Sensor Heads (SSH)

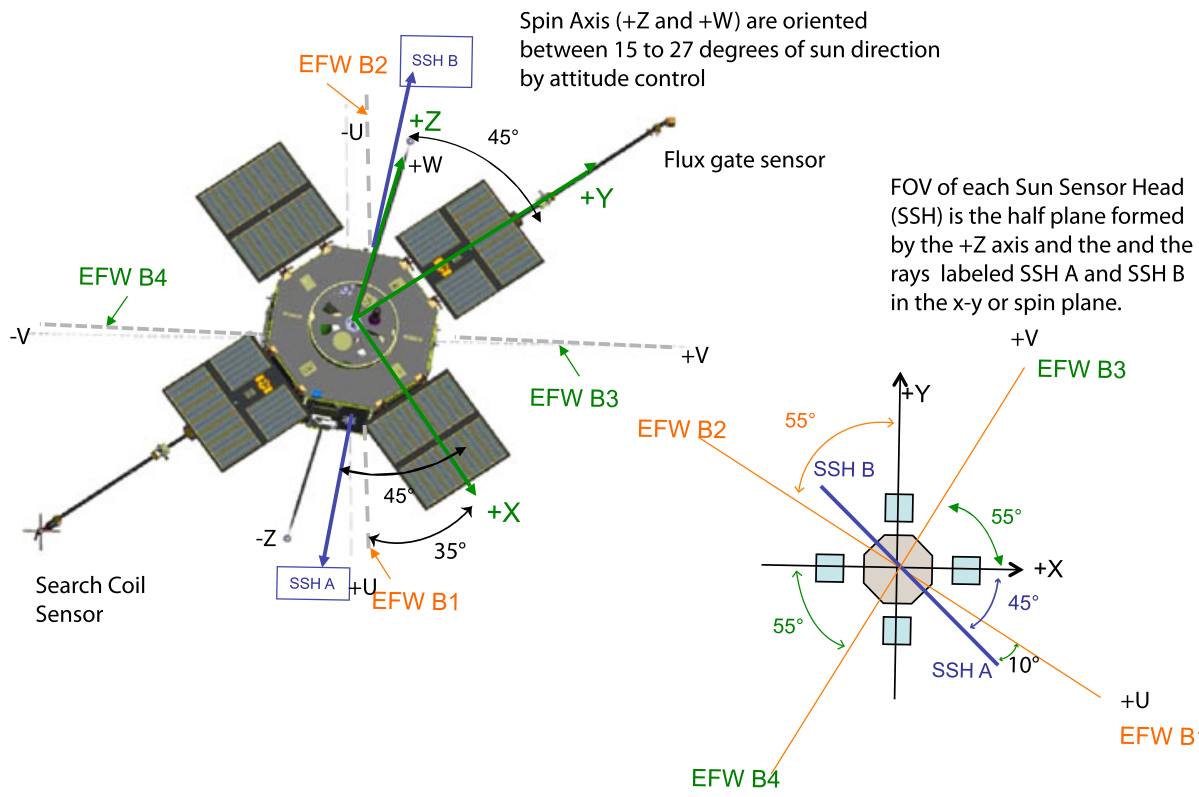

$+Z$ and + W out of plane towards sun

Fig. 7 Overview picture of RBSP spacecraft showing orientation of EFW spin plane boom sensors, EFW axial boom sensors, and EMFISIS 3-D Magnetic Search Coil sensors, spacecraft coordinates (xyz) and sensor coordinates (UVW) are indicated. Also shown are the two Sun Sensor Heads (SSH A and B) FOV directions relative to spacecraft coordinates and EFW spin plane booms. Magnetic Search Coil sensors directions and EFW booms are aligned in a common the UVW coordinate system for ease of analysis

Table 1 Boom locations and telemetry channels relative. To coordinate systems on the spacecraft

\begin{tabular}{llll}
\hline $\begin{array}{l}\text { EMFISIS Science } \\
\text { Coordinates (SCM\& }\end{array}$ & S/C & S/C-ICD & EFW TM \\
FGM) & Boom\# & Channel \\
\hline$+V$ & $+X+Y$ & 3 & 4 \\
$+U$ & $+X-Y$ & 1 & 2 \\
$-U$ & $-X+Y$ & 2 & 1 \\
$-V$ & $-X-Y$ & 4 & 3 \\
$+W$ & $+Z$ & 6 & 6 \\
$-W$ & $-Z$ & & 5 \\
\hline
\end{tabular}

directions of the electric field, flux gate magnetometer, and search coils. The three orthogonal search coil sensors and the three pairs of electric field probes are aligned with each other to facilitate comparison. Additional images of the spacecraft, coordinate systems, electric and magnetic field sensor booms, and instrument fields of view may be found in Kirby et al. (this issue).

The spacecraft rotates in a right-handed sense about the spacecraft $+\mathrm{z}$ axis with a nominal spin rate $\sim 5.5$ RPM. The spacecraft spin axis is directed between 15 and 27 degrees of the Sun, so that the spin plane booms rotate approximately in the $y-z$ GSE plane. The 


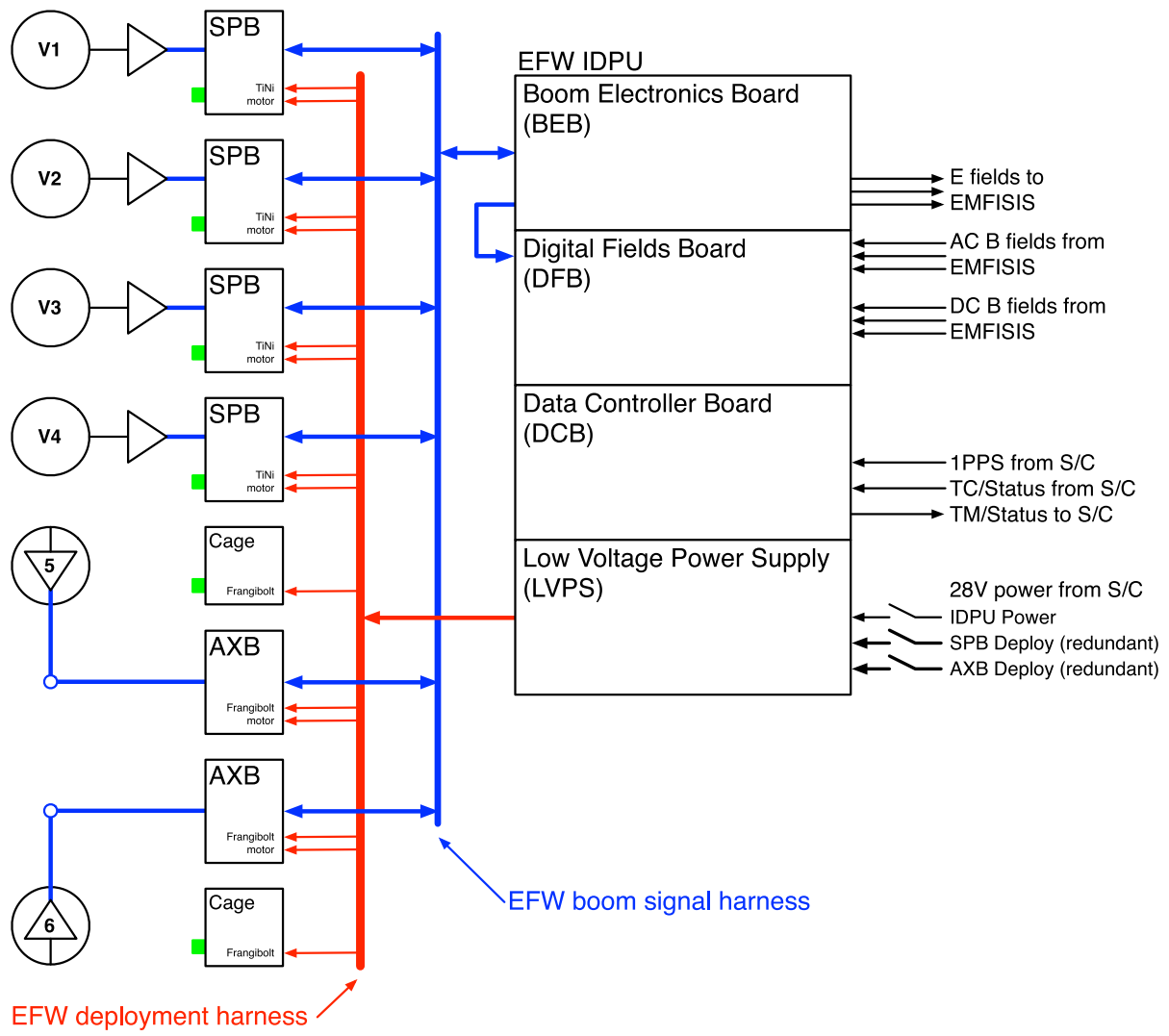

RBSP Electric Fields and Waves Instrument

Fig. 8 Block diagram of EFW instrument including Deployment Units, the EFW IDPU, and major interfaces with the spacecraft and with the EMFISIS instrument. The IDPU block shows major functional subsystems, as well as, signal lines (V1-V6), power lines, diagnostic lines (temperature, boom turns counters), and control lines (motor power, door controls) between the IDPU and the Deployment Units

electric field measurement is most accurate in this plane due to the length of the booms and the nearly uniform illumination of the sensors by the Sun.

A functional block diagram of the EFW instrument is presented in Fig. 8. Each EFW instrument consists of an Instrument Data Processing Unit (IDPU) and the six separate boom deployment units. The IDPU consists of a Boom Electronics Board (BEB), a Digital Fields Board (DFB), a Digital Control Board (DCB), and a Low Voltage Power Supply (LVPS) board. The EFW instrument transfers three high frequency analog differential electric field signals to the EMFISIS instrument (Kletzing et al. 2013, this issue). From EMFISIS, EFW receives three axis analog search coil AC magnetometer signals as well as the three axis fluxgate DC magnetometer signals. The EMFISIS search coil sensors are aligned with the electric field booms and are measured in the UVW coordinate system as illustrated in Fig. 7.

Table 2 presents a summary of the mass, power, and telemetry allocations of the EFW instrument and its major subsystems. 
Table 2 EFW mass. power, and telemetry

\begin{tabular}{llll}
\hline & Mass $(\mathrm{kg})$ & Power $(\mathrm{W})$ & TM \\
\hline IDPU & 6.4 & $11(@ 30 \mathrm{~V})$ & $12.0 \mathrm{kbits} / \mathrm{s}$ \\
Shielding & 2.2 & \multicolumn{2}{l}{} \\
$\begin{array}{l}\text { Spin Plane Booms } \\
(\times 4)\end{array}$ & $2.04(8.16)$ & - & - \\
$\begin{array}{l}\text { Axial Booms } \\
(\times 2)\end{array}$ & $3.12(6.24)$ & - & - \\
$\begin{array}{l}\text { Axial boom tube } \\
\text { assembly }\end{array}$ & 1.03 & - & - \\
Harness & 3.36 & - & - \\
Total & 27.39 & 10.2 & $12.0 \mathrm{kbits}$ \\
\hline
\end{tabular}

\section{EFW Design}

\subsection{Overview of Signal Path, Block Diagram and Science Quantities}

This section provides a brief overview of the signal paths and processing through the EFW instrument. A more detailed discussion of the design and functionality appears in subsequent sections.

The potential differences between each of the six spherical sensors and the spacecraft (labeled V1 through V6) are driven by unity gain preamplifiers near the sphere and the signals are sent down their respective boom cables to the Instrument Data Processing Unit (IDPU) where they enter the Boom Electronics Board (BEB). These signals are transferred directly to the Digital Filter Board (DFB) where AC coupled versions of the signals are generated in analog circuitry. The sensor signals from opposing booms are also differenced on the DFB board to provide AC (>10 Hz) and DC coupled versions of the three components of the electric field. The analog signals are digitized with 16-bit cross-strapped A-D converters on the DFB. Higher frequency $(10 \mathrm{~Hz}-400 \mathrm{kHz})$ wave electric field signals over the amplitude range $( \pm 40 \mathrm{mV} / \mathrm{m})$ are transferred to the EMFISIS wave instrument from the BEB via an analog buffer for wave analysis in that instrument. The digitized sensor and electric field measurements made on the DFB are transferred to the Digital Control Board (DCB) for formatting into Survey and Burst telemetry formats. The DFB provides the three components of the DC electric field from opposite sensors, denoted E12dc, E34dc, E56dc, which are sampled at $32 \mathrm{samples} / \mathrm{s}$ and digitally filtered at $10 \mathrm{~Hz}$ with a dynamic range of $\pm 1 \mathrm{~V} / \mathrm{m}$ and the single ended probe-spacecraft potential measurement (V1 through V6) sampled at 16 samples/s with a dynamic range of \pm 225 volts. These single sensor measurements when differenced on the ground can provide electric field measurements with a dynamic range up to $4 \mathrm{~V} / \mathrm{m}$. The waveform data are telemetered to the ground continuously over the entire RBSP orbit in a survey mode. The DFB also calculates for inclusion in the survey mode a variety of spectral and filter bank products. These include the complex Fast Fourier Transform (labeled SPEC) of selectable EFW field measurements over a frequency range of $1 \mathrm{~Hz}$ to $6.4 \mathrm{kHz}$. In the default mode, a spectrum is telemetered once every $8 \mathrm{sec}-$ onds and is organized into 64 pseudo-logarithmic frequency bins. The cross spectra between two selected measurements (XSPEC) is also calculated and telemetered to the ground in 64 pseudo-logarithmically spaced frequency bins. The two default measurements used in this calculation are the AC coupled differential electric field measurement from the 1-2 boom pair (E12ac) and the component of the search coil along the spin axis (SCM_W). Continuous 


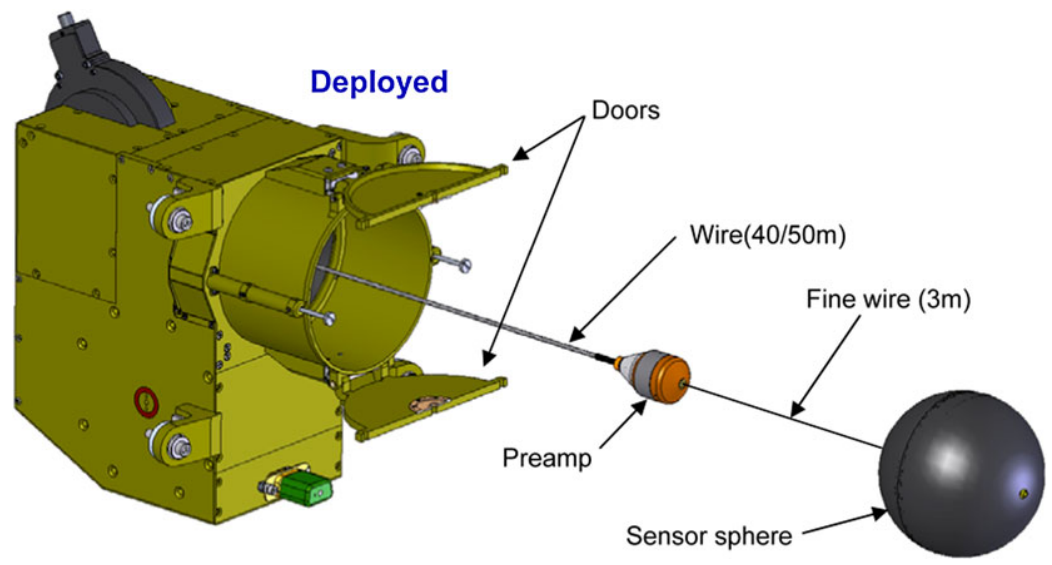

Fig. 9 EFW Spin Plane Boom Deployment Unit in deployed configuration showing deployment unit, cable, pre-amplifier housing, 3-meter thin wire, and spherical sensor

measurements of rapid variations in average power and peak values of waves, is provided in survey mode for two selectable quantities via a bank of broadband filters. These quantities are denoted FBK_1_av, FBK_1_pk, FBK 2_av, and FBK_2_pk respectively. In the default mode, these broad band filters are sampled at a cadence of 8 times per second in seven pseudo-logarithmically spaced frequency bins from $1 \mathrm{~Hz}$ to $6.5 \mathrm{kHz}$.

High time resolution measurements of waveforms, which cannot be continuously telemetered within the allocated EFW telemetry, are provided through two burst modes using two independent burst memories involving different programmable modes of collection and playback controlled by the DCB. The Burst telemetry nominally includes three high frequency versions of the differential electric field measurements, the six sensor-spacecraft potential measurements, and the three components of the search coil magnetometer. These modes will be described in more detail in later sections.

\subsection{Sensor System and Measurement Accuracy}

Spin Plane Sensors The spin plane sensor and cable system is illustrated in Fig. 9. The sensor is a conducting metal sphere of radius $4 \mathrm{~cm}$ coated with DAG 213 in order to minimize work function variations over the sphere and from sphere to sphere. In the deployed configuration, the sphere is connected to a high input impedance preamplifier via a thin $3 \mathrm{~m}$ long conducting wire. The sensors are current biased to control their floating potential and to minimize their sheath impedance. Theoretical calculations and comparison of measurements from biased and unbiased probes on ISEE-1, CRRES and Polar have shown that current biasing can reduce errors due to variations in floating potential by three orders of magnitude. In a manner similar to CRRES, Polar and Cluster, diagnostic sweeps in voltage will be used to determine the optimum value of the bias current. The sheath impedance coupling the electric field sensors to the plasma is adjusted through biasing to be $<10^{7} \mathrm{ohms}$ which is much smaller than the input resistance of the PMI OP-15 pre-amplifier input stage $\left(10^{11} \mathrm{ohms}\right)$. Similarly, the capacitive impedance of the 3-meter bare wire portion of the sensor dominates over the input impedance of the OP-15 preamplifier input stage. This sensor design dramatically limits both DC and AC voltage divider effects on input signals producing a near unity gain out to high frequencies. The frequency response of the electric field instrument ranges 
up to $400 \mathrm{kHz}$. The OP-15 was used on CRRES and on THEMIS. It is radiation tolerant to $50 \mathrm{krads}$ of total dose and is tantalum shielded to $100 \mathrm{krads}$ to meet the 2-year mission specification. The spin-plane boom cables carry power supply and biasing voltages out to the pre-amplifiers. They carry the sensor voltages measured by the pre-amplifiers back to the main electronics box.

An important error source in the measurement of electric fields is associated with the spurious photocurrents flowing between the spherical sensors and neighboring boom and sensor elements. These photocurrents produce fluctuations in the floating potential of the sphere surface relative to plasma potential. The small surface area of the very thin $3 \mathrm{~m}$ long wire connecting the sphere to the pre-amplifier is designed to limit the magnitude of such photo-currents to the sensor. In addition, the flow of photocurrents is controlled by voltage biasing of neighboring surfaces relative to the sphere potential. The biasing is controlled by the DCB microprocessor. The in-board surface called the guard is nominally biased at a constant value of $\sim 5$ volts negative relative to the sphere in order to limit the flux of photoelectrons to the sensor from the nearby cable and the large spacecraft surface. The outboard surface is typically biased $\sim 1$ volt negative to limit the outflow from the pre-amplifier housing to the sensor. Precise values will be determined through processor controlled bias sweeps and evaluation of the measurement accuracy at different points along the orbit during commissioning, as well as periodically during the mission.

The fact that the spin axis of the spacecraft is pointed nearly towards the Sun contributes to an especially sensitive electric field measurement in the spin-plane. This orientation results in nearly uniform solar illumination of the spin plane sensors over a spacecraft rotation. Consequently, the photocurrent to the spin plane sensors is also nearly constant over a spacecraft rotation. The electric field data will be less accurate when (1) the spacecraft is shadowed by the Earth so that the photo-currents necessary to produce a stable potential reference for the probes are not present, (2) during very brief periods when the thrusters on the spacecraft are firing and the spacecraft is surrounded by a thruster plume, (3) and during periods after attitude maneuvers when the boom cables are oscillating about their equilibrium points.

The spin-axis electric field is about an order of magnitude less accurate than the spin plane measurements since the spin axis booms are shorter by a factor of $\sim 7$ and have a comparatively greater error contribution from asymmetries in the spatial configuration of the spacecraft potential. A new feature of the spin-axis stacer booms on RBSP is that the length of the booms can be controlled such that, after on-orbit calibration, the sensors are positioned on the same equipotential of the spacecraft potential reducing offset errors to the electric field. Thus, the booms are not "popped" outward to a pre-determined length under the spring force of the stacer, but are restrained during deployment by the tension in the cable, which is slowly played out in a controlled fashion by a motor. The motor does not have a retract capability, but does allow the lengths of the two opposing spin axis booms to be independently adjusted.

Accuracy of the measurement along the spin axis booms is affected if the anti-sunward spin-axis boom is partially shadowed by one of the four solar panels as the spacecraft rotates. At these times, because there will be perturbations per spin period, the spin axis electric field component will be calculated from samples obtained from those intervals when the spin axis boom is not shadowed. In addition, spacecraft attitude is controlled by operators to minimize this shadowing by maintaining at least 15 degree offset of the spin axis from sun pointing.

On CRRES, during some of most intense electron injection events, the spacecraft differentially charged hundreds of volts relative to the plasma. These voltages exceeded the power supply rails of the pre-amplifier electronics and, as a consequence, the electric field 
instrument sometimes saturated during interesting electron acceleration events. On RBSP, the project instituted a rigorous program of electrostatic cleanliness to insure that the surface of the spacecraft was conducting and that conductive paths tied all major exterior surfaces together including the solar panels and thermal blankets. The electrostatic cleanliness program was designed to attempt keep spacecraft surfaces electrical equipotentials to within 1 volt and avoid differential charging.

The EFW instrument provides a measurement the spacecraft potential which can be used to estimate of the thermal plasma density in the plasmasphere and identify interval of spacecraft charging. The EFW instrument telemeters "single probe potentials", $\mathrm{V} 1 \mathrm{~s}, \mathrm{~V} 2 \mathrm{~s}, \ldots, \mathrm{V} 6 \mathrm{~s}$, which consist of the measured potential differences between individual probe and the spacecraft. The spacecraft potential is calculated on the ground by summing the single probe potentials from sensors on opposite sides of the spacecraft. This sum removes the differential electric signal due associated convection, waves, and other ambient plasma processes. In lower density cold plasmas $\left(<100 \mathrm{~cm}^{-3}\right)$, the spacecraft potential relative to the ambient plasma is primarily determined by the balance between photoemission associated with solar illumination and the thermal current associated with thermal electrons in the plasma. The current-biased probes provide a stable reference (within 1-2 volts of plasma potential) for the measurement of the spacecraft potential relative to the ambient plasma. Interpretation of the spacecraft potential in terms of the properties of the thermal electron plasma must include the fact that the spacecraft is typically in the Langmuir probe "focusing regime" (Pedersen 1995; Pedersen et al. 2008). In these circumstances the spacecraft potential scales as the logarithm of the electron density with a small contribution from temperature effects. The spacecraft potential is typically calibrated periodically against other measures of plasma density. As discussed in the Introduction, Fig. 6 presents a calibration of the spacecraft potential versus density as determined from measurements of the upper hybrid frequency by the EMFISIS instrument (Kletzing et al. 2013) over the density range from 1 to $10^{-3}$ during one orbit. The spacecraft potential measurements provide a higher time resolution $(\mathrm{DC}-100 \mathrm{~Hz}$ ), but less accurate, measurement of the density and thermal plasma structure than the one obtained from the upper hybrid frequency measurement. The spacecraft potential algorithm for calculating density is inaccurate during intervals of negative spacecraft charging induced by very strong fluxes of $10 \mathrm{eV}$ to $1 \mathrm{keV}$ electrons near apogee. Periods of strong spacecraft charging may be identified by comparing the spacecraft potential to the HOPE measurement of $\mathrm{H}+$ and $\mathrm{O}+$ over the $10 \mathrm{eV}$ to $5 \mathrm{keV}$ range (Reeves et al. this issue).

Sensor Frequency Response The frequency response of the spin-plane sensor system is governed by the properties of the plasma sheath around the sensors and by the ability of the preamplifier to drive the long 50-meter cables. The source impedance of the plasma sheath surrounding the sensor forms a voltage divider with the input impedance of the preamplifier system. The switch from resistive to capacitive coupling is associated with a roll-off in frequency response from unity near DC to 0.6 at $\sim 100 \mathrm{~Hz}$. A second higher frequency roll-off in the sensor/cable system occurs as a consequence of the capacitive loading of the preamplifier by the long spin-plane cables. This occurs near $300-400 \mathrm{kHz}$ and defines the upper end of the frequency response for the signals sent to the EMFISIS instrument.

Figure 10 presents plots of the gain and phase shift of the spin plane and spin axis boom pre-amplifier system and cables at the entrance to the EFW IDPU. Note that the spin axis booms have a higher frequency response as a consequence of the decreased attenuation due to the lower capacitance of the shorter spin axis boom. Additional phase shifts for these measurements are associated with the 5 pole Bessel anti-aliasing filters. For Bessel filters 
Fig. 10 Panel A. Calculated (for low density plasma) SPB sensor frequency response (including phase shift) of signals input to spherical sensors and output at entry to IDPU. Panel B: Same as Panel A for spin axis sensor. The first vertical green dotted line (near $8.2 \mathrm{kHz}$ ) indicates the highest frequency signal processed by the EFW instrument. The second dotted line at higher frequency indicates the required upper frequency of the signal passed to the EMFISIS wave instrument
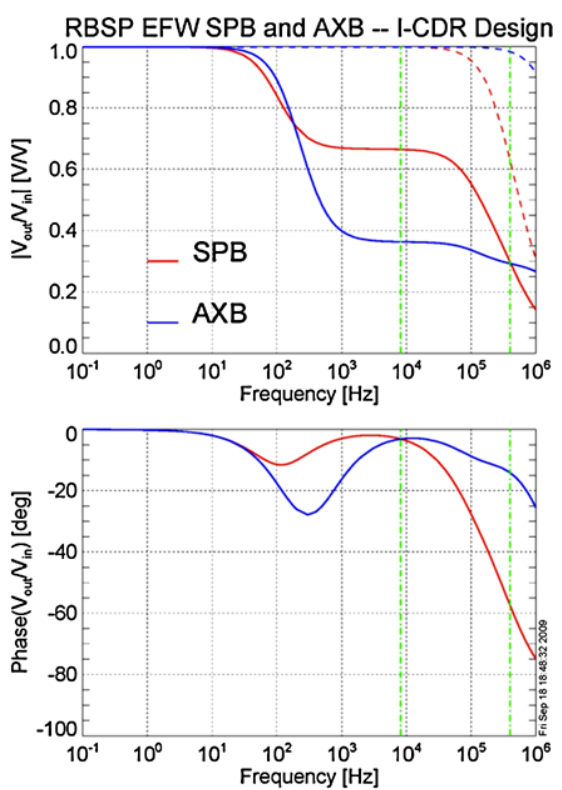

Table 3 Time delay due to FIR 5 pole Bessel filters

\begin{tabular}{cccc}
\hline Sampling rate & Delay $(\mathrm{ms})$ & Sampling rate & Delay $(\mathrm{ms})$ \\
\hline 1 & 6007.446 & 256 & 30.884 \\
2 & 3007.446 & 512 & 19.165 \\
4 & 1507.446 & 1024 & 13.306 \\
8 & 757.446 & 2048 & 10.376 \\
16 & 382.446 & 4096 & 8.911 \\
32 & 194.946 & 8192 & 8.179 \\
64 & 101.196 & 16384 & 7.813 \\
128 & 54.321 & & \\
\hline
\end{tabular}

the phase shift is linear as a function of frequency. For time domain signals this results in a constant time delay for a given roll-off frequency. Table 3 provides the time delays for different anti-aliasing frequencies for the 5 poles Bessel filters appropriate for all quantities in the burst and survey data.

On-orbit Calibration and Analysis of the Electric Field Measurement Calibrations of the electric field instrument can be provided in several ways. During quiet times when geophysical electric fields are small, the electric field measurement should approach $E=-V_{\mathrm{sc}} \times B$ where $V_{\mathrm{sc}}$ is the spacecraft velocity relative to the rest frame of the Earth and B is the measured magnetic field. For electric fields that are constant over a spin period, the measured signal from orthogonal booms pairs should be 90 degrees out of phase and scale in magnitude with $100 \mathrm{~m}$ boom lengths. During periods of strong flows in the near Earth plasma sheet, the velocity moments, $V_{P}$, from the HOPE plasma instrument can be used to estimate $E=-V_{\mathrm{P}} \times B$.

Historically it has been found that the most accurate quasi-static electric field determinations are provided by least-squares spin-fits to the electric field measured by the spin plane 
sensors in the rotating frame of the spacecraft. In the rotating system, a constant electric field in inertial space appears as a sinusoidal signal. This fit determines the optimum values of the amplitude and phase of this sinusoidal signal, determining the magnitude and direction of the electric field projected into the spin plane. Such spin fits are especially accurate because they use the large number (484) of measurement points gathered over one spin period and because they remove work function voltage differences between the probes and other errors which appear as constant offsets to the sinusoid. After one fit, the fit may be further optimized by removing noise points far from the fit value (i.e. more than 2 standard deviations) and repeating the process as needed to obtained the desired accuracy. Perturbations to the sinusoidal electric field signal observed by the rotating sensors due to angle dependent spurious photo-currents or wake effects can removed by selectively masking data points over those rotation angles most susceptible to the perturbations.

Analysis of data from the CRRES spacecraft, which also had a sun-pointing spin axis, shows that the dominant error source for the quasi-static spin plane electric field is typically due to the effect of attitude uncertainties in the Lorentz transformation from the spacecraft frame to the inertial frame of the Earth. The attitude uncertainty of $<3^{0}$ will allow EFW to meet or exceed the spin-plane boom measurement sensitivity requirement above $3 R_{\mathrm{E}}$ radial distance (either of $0.3 \mathrm{mV} / \mathrm{m}$ or $10 \%$ of the amplitude of the electric field, which ever is larger). The anticipated Spin plane electric field sensitivity after spin fits and other ground analysis efforts is expected to be $0.1 \mathrm{mV} / \mathrm{m}$ (or $10 \%$ of the amplitude) based on CRRES measurements (Rowland and Wygant 1998).

Spin Axis Electric Field Measurement The spin axis measurement is especially accurate for higher frequency wave measurements $(>100 \mathrm{~Hz}$ to $400 \mathrm{kHz})$ where it provides for a full three-dimensional electric field measurement. However, for quasi-static and low frequency measurements, the measurement is less accurate than the spin plane booms since the spin axis booms provide a shorter measurement baseline. In addition, the spin axis sensors are closer to the spacecraft and sample a larger fraction of the spacecraft charging structure especially at apogee where the Debye length is larger than the spacecraft dimensions and the charging structure has a slower fall-off with radial distance. Since this structure is asymmetric with respect to the Earth-Sun line, a portion of this charging structure appears as a differential signal in the low frequency electric field. These asymmetric contributions to the spin axis measurement are partially mitigated on RBSP, since the lengths of the individual spin axis booms may be incrementally adjusted (outward only) during on-orbit calibrations to remove the contribution from the spacecraft potential to the electric field. Finally, the spin axis booms may be shadowed by solar panels, which rotate and intermittently shadow the antisunward boom varying the photoemission and potentials of booms surfaces. This shadowing can produce periodic spikes repeating at the spin period lasting $<1 / 16$ second, which must be removed from the data. This photoemission variability is strongest for the anti-sunward spin-axis boom when the spin axis of the spacecraft points directly at the Sun. This shadowing near the electric sensors is mitigated on RBSP through on-orbit attitude maneuvers that control the angle between the spin axis of the spacecraft and the Earth sun-line so that it is always larger than 15 degrees. Measurement of the quasi-static spin-axis electric field requires extensive ground analysis and validation. The quasi-static spin axis electric field measurement is most useful for large electric fields associated with ULF waves, shocks, and injection events. In addition, it is designed to provide useful information on the structure of the largescale convection electric field during major geomagnetic storms when the field is especially strong and penetrates to lower L-value, and higher plasma densities (Wygant et al. 1998; Rowland and Wygant 1998). It is complemented when possible by the derivation of the spin 
axis electric field from the measurement of the two spin plane components of the electric field and the full three dimensional magnetic field measurement (from EMFISIS) and the constraint that (for large scale slowly varying fields) $E \bullet B=0$.

IDPU Electronics: Boom Electronics Board The signals from the six electric field sensors enter the IDPU onto the Boom Electronics Board (BEB). At this point, the DC signal has a gain of near unity and a dynamic range of $\sim 225$ volts. The sensor electric field signal is transferred to the Digital Filter Board (DFB) for signal processing and A-D conversion. Signals from opposing booms are subtracted and sent as buffered wave electric field signals with a frequency response up to $400 \mathrm{kHz}$ to the EMFISIS instrument. The required noise level of the signal to the EMFISIS instrument at the output of this buffer is $10^{-14} \mathrm{~V}^{2} / \mathrm{m}^{2} \mathrm{~Hz}$ at $1 \mathrm{kHz}$ and $10^{-17} \mathrm{~V}^{2} / \mathrm{m}^{2} \mathrm{~Hz}$ at $100 \mathrm{kHz}$. This was verified at EFW instrument level testing and also during interface testing with the EMFISIS instrument itself. The required minimum dynamic range is $\pm 30 \mathrm{mV} / \mathrm{m}$. This value is met and exceeded by the actual dynamic range of $\sim 40 \mathrm{mV} / \mathrm{m}$.

One of the principle functions of the BEB is to generate and transfer the different control voltages that are used for current biasing of the probe and to control the potential of surfaces near the sensors. These signals are transferred to each of the six boom deployment units and then out via wires in the boom cables to each of the sensors. These bias control circuits consist of the current bias circuit, the usher bias circuit, and the guard bias circuit. Each of the sensors is independently controlled by a set of bias circuits.

The current bias circuitry results in the injection of a microprocessor-controlled bias current from the sensor surface into the plasma to control the sensor floating potential and the plasma sensor-sheath resistance. This is achieved by setting the operational point on the current-voltage curve of the sensor/plasma sheath. In low-density plasmas, the bias current is generally adjusted to be a significant fraction of the total photocurrent to the probe. The injected bias current may be adjusted over a range between $\pm 500 \mathrm{nA}$ to an accuracy of $0.2 \%$ by the bias circuitry. The bias circuit has a high frequency roll-off $(6 \mathrm{~dB})$ at about $300 \mathrm{~Hz}$. The optimum bias current will be determined by on-orbit bias sweeps during the commissioning phase and also at a lesser cadence throughout the mission. Depending on on-orbit calibration results, the bias current may have different values in the low-density plasma near apogee and in the higher density plasma deep within the plasmasphere.

The usher and guard bias circuits generate voltages that equal the sum of a sphere output plus a microprocessor-controlled voltage offset. The roll-off for the usher signal is $300 \mathrm{~Hz}$ and that for the guard circuit is $100 \mathrm{~Hz}$. These circuits control the perturbing sensor-boom photocurrents over the frequency range from DC to somewhat less than $100 \mathrm{~Hz}$ where the sensor-plasma sheath impedance is resistive. The usher and guard voltages are transferred via the boom cable to their respective conductive control surfaces on the preamplifier sensor housing. Thus, the potential of these control surfaces relative to the sensor are a constant microprocessor-controlled value. The voltages may be adjusted to a constant value over a range of \pm 40 volts with a 16-bit DAC. The usher and guard bias potentials will be optimized during the commissioning phase and periodically throughout the mission. Typical values range between zero and several times the photoelectron e-folding energy or $\sim 5$ volts. Typically, once adjusted to their optimum values, the guard and usher voltages remain constant for months to years. The commanded values of the bias, guard, and usher voltages are included in EFW housekeeping telemetry, as are the parameters of the bias sweeps.

Search Coil Signals The three components of the wave magnetic field from the EMFISIS search coils (Kletzing et al. 2013) are provided to the EFW instrument via analog lines 
EFW MSC gain, phase - attenuated, bandwidth limited

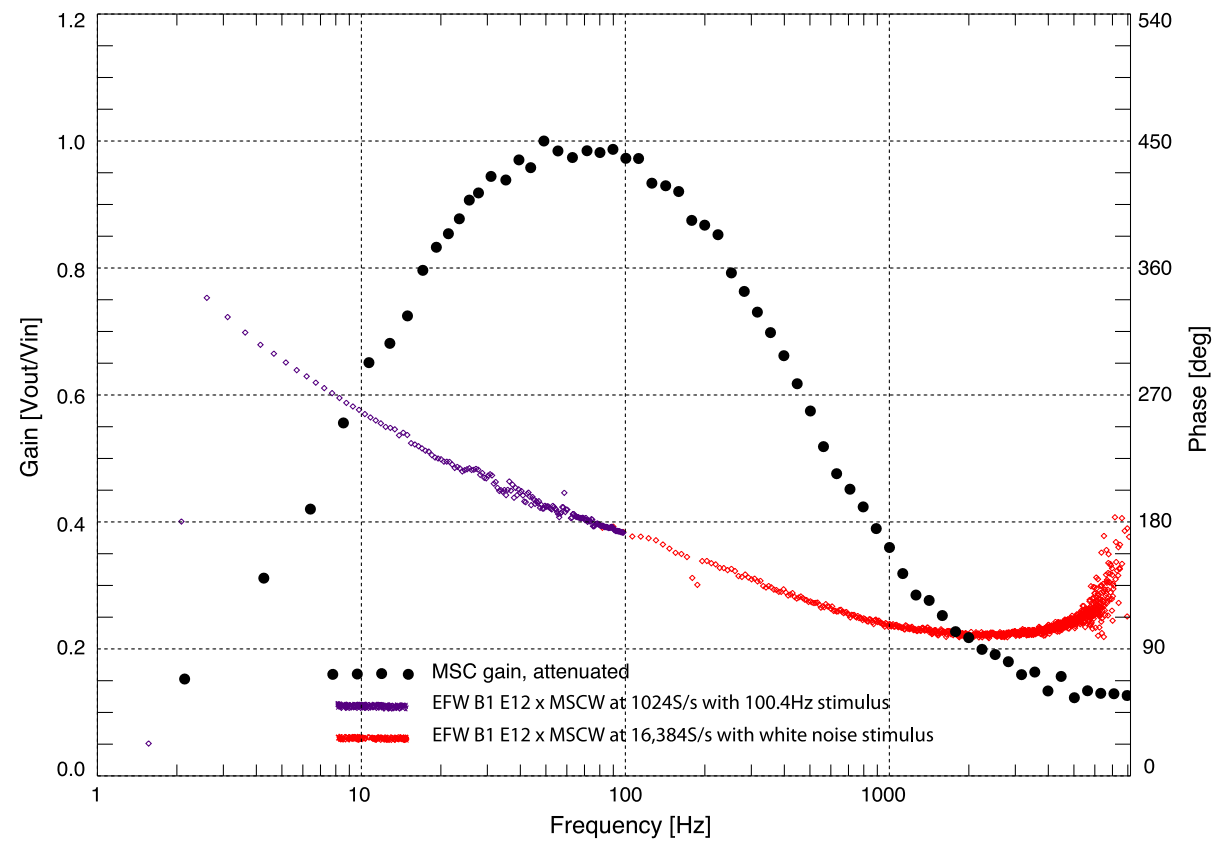

Fig. 11 Magnetic Search Coil gain and phase frequency response through EMFISIS search coil sensors to EFW telemetry from $2 \mathrm{~Hz}$ to $7 \mathrm{kHz}$. The different colored dots on the phase curve correspond to different signal generator modes driving the stimulus coils

where they are digitized along with the electric field signals. The EFW instrument science measurements focus primarily on the lower frequency $(<250 \mathrm{~Hz})$ portion of the wave signal. The maximum response for the EFW search coil signal is at about $100 \mathrm{~Hz}$ but on-orbit measurements indicate that large amplitude waves can be detected in the $>2 \mathrm{kHz}$ range. Search coil data is incorporated into EFW spectral products and also burst data collections. The gain (left hand label) and phase (right hand label) responses of the search coil were calibrated relative to the electric field signals during bench tests and also on the spacecraft. The results of these calibrations are shown in Fig. 11. The blue and red traces of the phase response simply refer to the nature of the signal from the signal generator that provided the spectral power for optimum calibration. A sinusoidal signal was used at low frequency (blue) and a broad band "white noise" signal was used for high frequency calibration (red).

\subsection{Digital Filter Board}

The Digital Filter Board (DFB) and associated firmware was designed, fabricated, and tested by a team from the Laboratory for Atmospheric and Space Physics (LASP) at the University of Colorado led by Robert Ergun. The digital filter board is responsible for analog processing of sensor signals, A-D conversion, and anti-aliasing, as well as onboard calculation of a variety of spectral and cross-spectral products.

Time Domain measurements As indicated by Fig. 8 diagram, and as previously discussed, the analog signals from each of the six spheres are passed to the DFB where the signals from 
Table 4 Survey mode time domain measurements (100\% duty cycle)

\begin{tabular}{llllll}
\hline Survey data & Range & $\begin{array}{l}\text { Resolution } \\
\text { (A-D) }\end{array}$ & $\begin{array}{l}\text { Samples/s } \\
\text { (Default) }\end{array}$ & $\begin{array}{l}\text { Duty } \\
\text { cycle }\end{array}$ & $\begin{array}{l}\text { TM } \\
\text { bits/s }\end{array}$ \\
\hline $\begin{array}{l}\text { E12S, E34S, } \\
\text { E56S }\end{array}$ & $\pm 1 \mathrm{~V} / \mathrm{m}$ & $30 \mu \mathrm{V} / \mathrm{m}$ & 32 & $100 \%$ & 1536 \\
$\begin{array}{l}\text { V1, V2, V3, } \\
\text { V4, V5, V6 }\end{array}$ & $\pm 225 \mathrm{~V}$ & $6 \mathrm{mV}$ & 16 & $100 \%$ & 3072 \\
\hline
\end{tabular}

opposing boom pairs are differenced and filtered in analog circuitry to provide three analog signals proportional to the electric field. In addition, higher gain AC coupled versions of the analog signals are generated to more sensitive wave measurements.

The analog filtering roll-off is performed by 5 pole Bessel filters with a linear phase shift with frequency. This linear phase shift in frequency space corresponds to a constant time delay for signals in the time domain and gives an undistorted waveform.

After A-D conversion, signals are digitally filtered with 5 pole low pass Bessel filters at an adjustable Nyquist frequency. The Bessel filters have a near linear phase shift as a function of frequency. This results in a constant group delay over almost the entire bandpass, which minimizes distortion of the waveform of the analog signal. This property is particularly useful when performing interferometric timing between opposite probes. The digital filtering is implemented in FPGA based firmware. The filtering algorithm consists of a recursive algorithm that allows filtering with programmable $\left(2^{N}\right)$ Nyquist frequencies. The maximum filter frequency is $6.5 \mathrm{kHz}$. The total group delays for different sampling frequencies are presented in Table 3.

There are three contributions to phase shifts between the "natural" and telemetered electric field waveforms. One is the phase shift due to the sensor-plasma sheath, one is the sensor-cable frequency response/phase shift, and the other is associated with the antialiasing filters of the DFB board. The first two contributions are shown in Fig. 10. The last contribution consisting of the constant time delays, due to the linear phase shift of the analog 5 pole Bessel filters set at $6.5 \mathrm{kHz}$, and also the time delays due to the FIR 5 pole Bessel filters discussed above and presented in Table 3 should be included during ground analysis.

The higher frequency digitized electric field waveforms (up to $16.4 \mathrm{k}$ samples/s) are transferred to the Data Controller Board (DCB) for waveform recordings in either of two burst memory systems. The burst data sampling rates, duration of the burst, and selection of specific time intervals to record into memory, as well as, which of the intervals in memory to telemeter to the ground, are all controlled by the microprocessor in the DCB.

The DFB produces the three components of the 16-bit digitized electric waveform sampled at 32 samples/s (E12_SVY, E34_SVY, and E56_SVY). It provides six single-ended measurements of the potential difference between the individual electric sensors and the spacecraft sampled at 16 samples/s (denoted V1_SVY through V6_SVY). These quantities are described in Table 4. In addition, it provides higher time resolution data inputs for either of the two instrument burst modes. The burst 1 mode includes three components of the electric field (E12_B1, E34_B1, E56_B1), six components of the spacecraft-sensor potential (V1_B1 through V6_B1), and three components of the AC magnetic field from the EMFISIS search coil magnetometer (SCM_U_B1, SCM_V_B1, SCM_W_B1). The burst 2 mode data returns a similar complement of electric field (E_12ac_B2, E34ac_B2, E56ac_B2), search coil (SCM_B2, SCM_2B2, and SCM_B2) and single ended measurements (V1ac_B1 through V6ac_B2) with the exception that in the default mode the single ended and electric field signals are AC coupled with a higher gain. 
Table 5 Survey mode spectral and cross spectral products (100\% duty cycle)

\begin{tabular}{llllll}
\hline $\begin{array}{l}\text { Default data } \\
\text { selection }\end{array}$ & $\begin{array}{l}\text { Default \# } \\
\text { Comp }\end{array}$ & Range & $\begin{array}{l}\text { Freq. } \\
\text { Bins }\end{array}$ & $\begin{array}{l}\text { Samples/s } \\
\text { (Default) }\end{array}$ & $\begin{array}{l}\text { TM } \\
\text { bits/s }\end{array}$ \\
\hline $\begin{array}{l}\text { Spectral Power: } \\
\text { E12ac E56ac, }\end{array}$ & 4 & $100 \mathrm{~dB}$ & 64 bins & $1 / 8$ & 448 \\
$\begin{array}{l}\text { SCMpar, SCMprp. } \\
\text { Cross Spectra }\end{array}$ & 2 & $\begin{array}{l}\text { Phase } \\
\text { SCMW } \times \text { E12ac, }\end{array}$ & 64 bins & $1 / 8$ & 768 \\
\begin{tabular}{l} 
V1ac $\times$ V2ac \\
\hline
\end{tabular} & & difference & & & \\
\hline
\end{tabular}

The DFB receives signals from the EMFISIS search coil magnetometer via an analog interface to provide measurements of the 3-D wave magnetic field. These data may be used as input to the EFW spectral products or filtered and sent to the DCB to be included in EFW time domain burst recordings.

The DFB also receives DC fluxgate magnetometer analog signals from the EMFISIS instrument in order to allow for rotation of EFW data into a magnetic field aligned coordinate system on-orbit. This data stream is not normally included in EFW telemetry. However, for purposes of redundancy, the digitized fluxgate data can be included in EFW telemetry in the event of failure of the EMFISIS digital section. This redundancy is motivated by the important role that the DC magnetometer data play in the RBSP spacecraft attitude determination and analysis of field aligned coordinates for the different science instruments. There is a similar redundant data path for EFW electric field data above $10 \mathrm{~Hz}$ via the EMFISIS instrument. The EFW preamplifiers and signal buffers to the EMFISIS instrument will remain powered in the event of a failure of the EFW digital section.

Survey Mode Spectra and Cross Spectra In the survey mode, the DFB provides a continuous stream of electric and magnetic field spectra over the frequency range of $\sim 1 \mathrm{~Hz}$ to $6.5 \mathrm{kHz}$ at a cadence of one spectrum every 8 seconds. It calculates 2048-point complex FFT spectra and cross spectra every 0.125 second over a frequency range from $1 \mathrm{~Hz}$ to $6.5 \mathrm{kHz}$. Prior to the FFT calculation, the waveform data are multiplied by a Hanning window. Subsequent to the FFT calculation, in order to save telemetry, the real (R) and imaginary (I) parts of FFT frequency components are pseudo-logarithmically compressed into 36, 64 or 112 frequency bins. In the default mode, the spectra accumulated over 1/8 second are averaged together over an 8 second period to provide the real and imaginary components of selected wave forms in 64 frequency bins every 8 seconds. The quantities that can be selected for the spectra and cross-spectra are presented in Table 5.

In addition, the complex cross-spectra are calculated for four selected pairs of quantities. This allows us to calculate, on the ground, spectra of the estimated complex E/B ratio, the magnetic field-aligned Poynting flux, wave polarization, or, the correlation (or anti-correlation) between density and the magnetic field magnitude fluctuations. These calculations are especially designed to routinely determine the properties of whistler waves which provide strong signal to noise ratios and have pulse widths nearly comparable to the $125 \mathrm{~ms}$ spectral sampling interval The results of these calculations can be compared for brief selected periods of time to similar calculations using the full three dimensional electric and magnetic field obtained from the burst recordings. The number of frequency bins, the sampling interval may be optimized on the basis of these comparisons.

The DFB board uses a CORDIC algorithm to rotate wave electric and magnetic field data into a magnetic field aligned coordinate system based on the direction of the average magnetic field. The rotation is performed in the spin plane of the spacecraft to obtain $E_{\text {perp }}$, which 
Table 6 Survey mode: broad band filters banks (FB) (100\% duty cycle)

\begin{tabular}{|c|c|c|c|c|c|}
\hline & $\begin{array}{l}\text { Default } \\
\text { quantities }\end{array}$ & $\begin{array}{l}\text { Freq. Bins } \\
\text { (default) }\end{array}$ & Range & Sensitivity & $\begin{array}{l}\text { Cadence } \\
\text { (samples/s) }\end{array}$ \\
\hline Power & $\begin{array}{l}\text { FB1: } \\
\text { E12dc aver }\end{array}$ & $\begin{array}{l}0.8 \mathrm{~Hz}-6.7 \mathrm{kHz} \\
\text { (7 log bins) }\end{array}$ & $1 \mathrm{~V} / \mathrm{m}$ & $20 \mu \mathrm{V} / \mathrm{m}$ & 8 \\
\hline Power & $\begin{array}{l}\text { FB2 } \\
\text { SCMa_ave }\end{array}$ & & & & 8 \\
\hline $\begin{array}{l}\text { Peak } \\
\text { value }\end{array}$ & $\begin{array}{l}\text { FB1: } \\
\text { E12dc_peak }\end{array}$ & & & & 8 \\
\hline $\begin{array}{l}\text { Peak } \\
\text { value }\end{array}$ & $\begin{array}{l}\text { FB } 2 \text { SCMa } \\
\text { peak }\end{array}$ & & & & 8 \\
\hline
\end{tabular}

is the component of the electric field perpendicular to the magnetic field direction in the spin plane and $E_{\mathrm{par}}$ or the component of the electric field closest to parallel to the magnetic field within the spin plane. The spin axis electric field component is not included in the rotation. The wave magnetic field data is also rotated in order to determine the projection of the wave magnetic field orthogonal to the background magnetic field direction and orthogonal to the direction of $E_{\text {perp. }}$. The data from this field-aligned coordinate system may be input into the spectral or cross spectral products in survey mode to provide spectral information on wave Poynting flux parallel to the average magnetic field and also the velocity of propagation of waves along the magnetic field. CORDIC rotated data may also be incorporated in burst formats for playback to the ground. A cross-correlation algorithm allows calculation of the phase-lag between opposite boom pairs, which can provide information on the velocity of propagation of small-scale plasma structures propagating from one sensor to the opposing sensor with a baseline separation of 100 meters. This technique is most feasible for structures with scale sizes of several kilometers or less and travelling at less than $800 \mathrm{~km} / \mathrm{s}$. The fidelity of these calculations will be determined by comparison to the full three dimensional electric and magnetic field wave forms which are downloaded from burst mode recording.

Survey Mode Filter Bank Data The DFB also provides measurements of the average power and peak amplitudes from either 7 (default) or 13 logarithmically spaced band-pass filters, implemented in FPGA based firmware, between $0.8 \mathrm{~Hz}$ and $6.5 \mathrm{kHz}$ sampled at a cadence of 8 samples/s. Details of the filter banks are summarized in Table 6. Two quantities may be input into the filter banks. In the default mode, V12ac, the AC coupled measurement of the electric field from sensors 1 and 2 in the spin plane is input into filter bank 1 And SCMw, the spin axis component of the search coil magnetic field serves as input into the second filter bank. The broadband filters provide a continuous stream of survey mode measurements over the entire orbit providing information on rapid time variations and bursty wave activity over a broad frequency spectrum. These measurements are also used as an input to burst trigger algorithms.

\subsection{Data Controller Board and Burst Memory Modes}

The Data Controller Board (DCB) is responsible for the reception of instrument commands, timing and status information from the spacecraft command and data handling system. The DCB implements commands, keeps track of internal data acquisition timing and monitors spacecraft status. It controls instrument data compression, telemetry formatting and sends all EFW telemetry to the spacecraft for transmission to the ground. It controls instrument operational modes including bias sweeps on the BEB board. It also handles burst memory 
sampling formats, triggering, recording, playback, and memory management for the DFB. It controls all boom deployments. It calculates space weather products including electric field spin period sine wave fits and the spacecraft potential. It also controls the redundant magnetometer back-up mode in the event of an EMFISIS instrument failure.

The DCB is based on a general purpose microprocessor, the Z80, an 8-bit processor implemented as FPGA firmware. The FPGA chosen for flight is an Actel RTAX2000S. It includes a Z80 processor as an instantiation of the CAST Inc. Z80 IP-core. The CPU in the FPGA is supported by external $32 \mathrm{~K} \times 8$ boot PROM, $128 \mathrm{~K} \times 8$ SEU-immune static RAM and $128 \mathrm{~K} \times 8$ EEPROM. Additional logic within the FPGA handles the processor bus control and provides registers for accessing the various sections of memory. Also included in the FPGA are the instrument interfaces, the SDRAM controller, the FLASH memory controller, error detection and correction logic for SDRAM and FLASH memories, the spacecraft interface logic, DMA and data management control, analog housekeeping control, and timing/time-tagging support.

Like the other EFW IDPU boards, the DCB resides on a 6U VME board, connecting to a custom instrument backplane. Power is received through the backplane connection, which is also used to communicate with the other IDPU boards: the LVPS, the BEB and the DFB (described elsewhere). The DCB receives its operating power from the LVPS as well as a number of analog housekeeping values. The DCB controls the boom deployment power switching in the LVPS. The DCB controls sensor biasing and modes in the BEB. The DCB controls the operating modes of the DFB and directs DFB data products via DMA to DCB memory.

The DCB serves as the EFW interface to the spacecraft command and data handling system. The interface includes a UART based telemetry interface (115.2 KBaud), a timing signal (1 Hz Clock and "Spin Pulse"), and a UART (115.2 Kbaud) based command interface.

For Burst-1 data storage, 32 GBytes of non-volatile FLASH memory is installed on the DCB. This bulk memory is composed of eight 4 GByte memory modules. Each memory module includes eight $512 \mathrm{M} \times 8$ Micron FLASH Memory devices. Each of the 4 GB modules is separately powered. FPGA based logic is used to streamline the intensive DMA data-transfer operations between FLASH and the DCB-based random access memory banks including error detection and correction. The CPU is responsible for continuous FLASH memory module management.

For Burst-2 data storage, 256 MBytes of local dynamic RAM memory is installed on the DCB. The SDRAM can be powered on and off to save power and clear problems. FPGA logic manages the SDRAM refreshing.

\subsubsection{Burst Memory and Operations}

The EFW instrument has two independent burst memory systems, which focus on different aspects of high frequency waves and involve different modes of data selection. The measurement quantity formats, data rates, modes of burst playback and triggers are programmable and are controlled by the DCB. Analog filtering, analog to digital conversion, and digital anti-aliasing occur on the DFB. The solid-state burst memories are located on the DCB. Tables 4-7 present the format, sampling, signal ranges, and A/D resolution of science quantities for burst 1 and burst 2 modes respectively.

Burst Mode 1 The Burst-1 mode uses the 32 GB flash memory for the recording and playback of high time resolution waveform data. Telemetry quantities and sampling rates are summarized in Table 7. In the default mode, the sampled quantities include three components of the DC coupled electric field, three components of the search wave magnetic field 
Table 7 Burst 1 data (16 bit quantities)

\begin{tabular}{|c|c|c|c|c|c|c|}
\hline \multirow{2}{*}{$\begin{array}{l}\text { Measurements } \\
\text { (nominal) }\end{array}$} & \multirow{2}{*}{$\begin{array}{l}\text { Meas. } \\
\text { Range }\end{array}$} & \multirow{2}{*}{$\begin{array}{l}\text { Meas. } \\
\text { Res. }\end{array}$} & \multicolumn{2}{|c|}{ Sample/s } & \multirow{2}{*}{$\begin{array}{l}\text { Duty } \\
\text { cycle }\end{array}$} & \multirow{2}{*}{$\begin{array}{l}\text { Bits/s } \\
\text { orbit Avg. }\end{array}$} \\
\hline & & & Max & Default & & \\
\hline $\begin{array}{l}\text { Electric field E12dc, } \\
\text { E34dc, E56dc }\end{array}$ & $\pm 1 \mathrm{~V} / \mathrm{m}$ & $30 \mu \mathrm{V} / \mathrm{m}$ & $16.4 \mathrm{k}$ & 512 & $4.00 \%$ & 983 \\
\hline $\begin{array}{l}\text { Sensor-SC potential: } \\
\text { V1dc, V2dc, V3dc, } \\
\text { V4dc, V5dc, V6dc }\end{array}$ & $\pm 225 \mathrm{~V}$ & $6 \mathrm{mV}$ & $16.4 \mathrm{k}$ & 512 & $4.00 \%$ & 1966 \\
\hline $\begin{array}{l}\text { Search coil } \\
\text { magnetometer } \\
\text { SCMU, SCMV, } \\
\text { SCMW }\end{array}$ & $12 \mathrm{nT}$ & $0.36 \mathrm{pT}$ & $16.4 \mathrm{k}$ & 512 & $4.00 \%$ & 983 \\
\hline Total telemetry & & & & & & 3932 \\
\hline
\end{tabular}

from the EMFISIS search coil, and the 6 values of the potential differences between each of the 6 sensors and the spacecraft. All these quantities are sampled at 512 samples/s in and low pass filtered at $\sim 200 \mathrm{~Hz}$ with a constant time delay 5 pole anti-aliasing filter in the Burst-1 default state.

An alternative format that could be selected includes ac-coupled versions of the signals with higher gains.

The sampling rate is programmable and with selectable values ranging between the default of $1 \mathrm{sample} / \mathrm{s}$ to $16.4 \mathrm{ksamples} / \mathrm{s}$ in factors of two increments with adjustable low pass anti-aliasing filters near the $80 \%$ of the Nyquist frequency or $6.4 \mathrm{kHz}$ at the highest sampling rate of $16.4 \mathrm{k}$ samples/s.

At the nominal rate with continuous sampling, the 32 GB memory would be filled in $\sim 20$ days. The telemetry allocation for playback is 3932 bits/s. This allows for about $4 \%$ of the orbit averaged total non-compressed burst 1 data to be played back. This corresponds to about 40 minutes of data at the default Burst-1 sampling rate. The minimum size burst is 2 Mbytes or about 5 minutes in the default mode. Higher sampling rates may be used and the corresponding fraction of the data played back is less.

Data compression algorithms implemented in the DCB flight software increase the actual volume of B1 and B2 recorded data played back on orbit by factor of 2-4.

The selection of the "most interesting" time intervals for high time resolution bursts and their relation to time intervals of strong particle energization and to intervals when high time resolution data are not obtained is of crucial importance to the EFW science goals.

In the default Burst-1 playback mode, the time intervals of the Burst-1 mode data to be returned to the ground for analysis may be selected by designated EFW scientists at the EFW SOC and uplinked to the EFW instrument for playback of Burst-1 data intervals on subsequent passes. This "human intervention" allows EFW scientists to identify interesting time intervals on the basis of EFW survey data and any data available from other instruments on the RBSP spacecraft. In addition, information from ground based investigations, other spacecraft, or the BARREL investigation will be incorporated into the burst selection decision as available.

Alternatively, the time intervals of the Burst-1 playback data may be autonomously determined by software algorithms in the DCB flight software. Some of the information from the EFW instrument to be used to decide the burst intervals of the most value include the DC electric and magnetic fields, information on intervals of intense wave activities using 
Table 8 Burst 2 data (16 bits)

\begin{tabular}{llllll}
\hline $\begin{array}{l}\text { Measurements } \\
\text { (nominal) }\end{array}$ & $\begin{array}{l}\text { Meas. } \\
\text { Range }\end{array}$ & $\begin{array}{l}\text { AD } \\
\text { Resol. }\end{array}$ & $\begin{array}{l}\text { Cadence } \\
\text { sample/s } \\
\text { (nominal) }\end{array}$ & $\begin{array}{l}\text { Duty } \\
\text { cycle }\end{array}$ & $\begin{array}{l}\text { Bits/orbit } \\
\text { (average) }\end{array}$ \\
\hline E12AC, E34AC, E56AC & $\begin{array}{l} \pm 400 \\
\mathrm{mV} / \mathrm{m}\end{array}$ & $12 \mu \mathrm{V} / \mathrm{m}$ & $16.4 \mathrm{k}$ & $0.100 \%$ & 786 \\
V1-V6 & $\pm 12.5 \mathrm{~V}$ & $0.4 \mathrm{mV}$ & $16.4 \mathrm{k}$ & $0.100 \%$ & 1573 \\
SCMU, SCMV, SCMW & $\pm 11 \mathrm{nT}$ & $0.36 \mathrm{pT}$ & $16.4 \mathrm{k}$ & $0.100 \%$ & 786 \\
Total & & & $0.100 \%$ & 3146 \\
\hline
\end{tabular}

spectral data and also power in electric fields and magnetic fields measured by broad-band filters which are sampled at 8 samples/s.

Candidate burst intervals of special interest include: (1) Waves observed during substorm injections and dipolarizations; (2) Waves observed during interplanetary shocks impacts on the magnetosphere; (3) Waves observed during observations of energetic electron microburst and other intervals of electron flux enhancements or loss revealed by the RBSP ECT MAGEIS high time resolution measurement of energetic electrons; (4) Intervals of magnetic conjunction with the BARREL balloon campaign designed to investigate microbursts and other modes of electron precipitation and loss; (5) Periods of high wave activity when the two RBSP spacecraft are "closely spaced" sampling the same wave field; and, (6) Science targets of opportunity including compressed front-side magnetopause crossings during major storms when energy transfer into the magnetosphere is the largest.

Burst Mode 2 The second burst waveform mode (Burst- 2 or interferometric mode) in the default mode typically samples each of the six individual electric field sensors at nominal rate of $\sim 16.4 \mathrm{ksamples} / \mathrm{s}$. Telemetry formats and sampling options for this mode are summarized in Table 8 . The signals from the individual probes provide interferometric timing information of small scale structures as they move over the spacecraft. These measurements allow the determination of the direction and velocity of propagation of the structure. Similar bursts have proven useful in determining the properties of ion phase space holes and other small scale structures (Ergun et al. 2001; Dombeck et al. 2001; Cattell et al. 2001) as they propagate along the magnetic field.

Burst-2 data collection is autonomously triggered with an algorithm in flight software on the DCB that continuously evaluates a quality factor which is a weighted linear combination of a variety of measured wave electric and magnetic field parameters. The most important of these parameters are the measured power and the peak in the electric and magnetic fields in the DFB broadband filters. The weights for the different field parameters used to compute the quality factor are stored in look-up tables that can be updated by ground command. Similar automated burst trigger algorithms have been used by electric field instruments on the FAST, Polar, Cluster, and THEMIS missions.

Burst-2 data are stored in $256 \mathrm{MB}$ of rad-hard SDRAM memory. In the EFW default telemetry mode, this burst mode can playback data covering $0.1 \%$ of the orbit period or for about 300 seconds without data compression. The burst information is played back in a prioritized list based on the value quality factor. This insures that the "best" events are telemetered to the ground. Burst 2 data can also be collected and played back on the basis of time tagged commands. 


\subsection{Low Voltage Power Supply Board}

The function of the Low Voltage Power Supply (LVPS) board is to generate voltages for the analog and digital sections of the IDPU and to controlling boom deployment motors. Receiving an unregulated supply voltage from the spacecraft power bus, the LVPS provides stable fixed (referenced to signal ground) voltages to power analog and digital circuitry throughout EFW. It also produces \pm 225 volts for the floating ground reference and \pm 15 volts floating power for the sensor pre-amplifiers. In addition, the LVPS receives two additional switched power lines from the spacecraft power bus to allow for deployment of the EFW mechanisms. When the spacecraft activates these primary circuits, the EFW DCB controls power switches within the LVPS to pop pin-pullers, fracture frangibolts and activate deployment motors.

The dynamic range of the electric field measurement in the spin plane must accommodate voltage swings that include the amplitude of the largest anticipated electric fields $(1 \mathrm{~V} / \mathrm{m})$ integrated over the boom separation distances $(50 \mathrm{~m})$ plus the largest (slowly varying) floating potential of spacecraft that is expected in low density plasmas (40 volts) or during electron injection events ( $<10$ volts). This motivates the use of a floating power supply system in which the sensor pre-amplifiers power supplies ( \pm 15 volts) are referenced to a floating ground reference driven by a low pass $(<500 \mathrm{~Hz})$ signal at the potential of the pre-amplifier output. This allows low frequency excursions of \pm 225 volt power supply which track the sphere outputs for potential variations below $500 \mathrm{~Hz}$. Higher frequency signals are tracked by the \pm 12 volt power supply.

This power supply system is used to power the pre-amplifiers at the ends of the booms. Special care in LVPS layout has been taken to insure that the power supplies are especially low in noise. This includes placement of traces and ground planes. The power converter frequency is fixed at $200 \mathrm{kHz}$ so that noise is generated only at this frequency and its integer multiples.

The power supplies for the sensor pre-amplifiers and the buffers to the EMFISIS system are designed to continue to function in the event of a failure of the EFW IDPU digital section. (As discussed, similar precautions have been taken by the EMFISIS team to provide a backup analog data stream for the fluxgate magnetometer data via the EFW instrument).

Spin Plane Booms and Deployment Units The electric field spin plane boom deployment units were designed, constructed and tested by the Space Science Laboratory at the University of California at Berkeley. Members of the boom engineering team have been responsible for the booms on the Air Force S3-3, ESA GEOS 1 and 2, NASA/ESA ISEE-1, Swedish Viking, Swedish Freja, NASA/USAF CRRES, NASA Polar, FAST, and THEMIS, and ESA/NASA Cluster spacecraft, as well as boom systems on dozens of sounding rockets.

The RBSP spin plane booms consist of two pairs of centripetally deployed booms in the spin plane of the spacecraft. As shown in Fig. 9, the booms are terminated with spherical sensors, which are separated by 100 meters. The boom deployment units are mounted on the periphery of the spacecraft at 90 degree intervals. In the stowed configuration, the spin plane boom cable is wound around a spool within the deployment unit and is deployed under the influence of the "centrifugal force" associated with the spacecraft rotation. The mass of one of the spin plane boom deployment units including sensor and cable is $\sim 2.0 \mathrm{~kg}$. The linear mass density of the boom cable is $\sim 3.6 \mathrm{gm} / \mathrm{m}$. Each deployment unit contains a rotating wire storage spool, a metering wheel based cable deployment assembly, a DC brush motor, over tension and end of wire indicators. A micro-switch is pulsed on each turn of the cable spool allowing flight software in the DCB to monitor and control the deployed length and to match the deployed lengths of opposing pairs of booms. 
Fig. 12 EFW Spin Axis Booms Deployment Unit in stowed and deployed configurations showing deployment assist device, stacer element, hinge and whip, and spherical sensors and cable

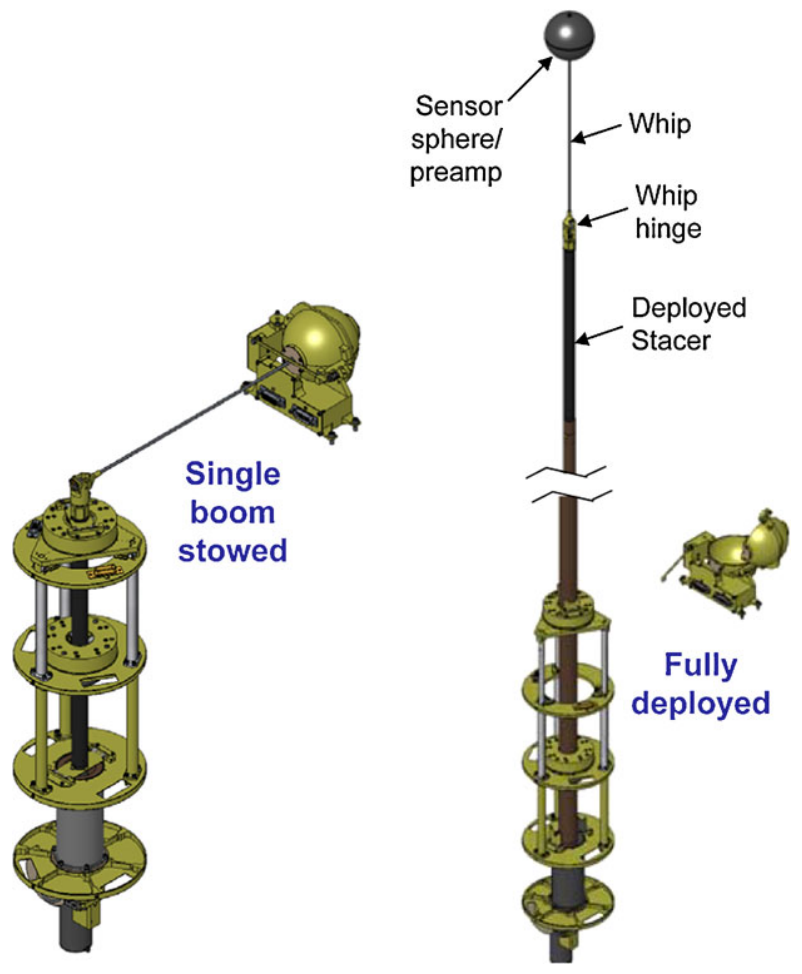

At the end of the deployed multi-conductor cable, a preamp enclosure is attached and contains the preamp electronics board, as well as acting as two of the photoelectron control surfaces (usher and guard). A fine wire exits the preamp housing and connects to a springloaded spool enclosed within a spherical probe of radius $4 \mathrm{~cm}$. Three meters of fine wire are wound onto the spool mechanism inside the spherical probe and the wire is intended to deploy by centrifugal force as the boom elements are deployed.

The motor in the base of each unit is shielded with mu metal to limit leakage of stray magnetic fields. Magnetic shielding is external to the motor, and does have some effect on performance, by its magnetic field short-circuiting effects. The drive mechanism is fully enclosed to provide EMI shielding, as well as to keep debris out. EMI filters are used on both power lines.

Spin Axis Booms The electric field axial boom deployment units were designed, constructed and tested by the Space Science Laboratory at the University of California at Berkeley. Members of the boom engineering team have been responsible for the booms on the Air Force S3-3, ESA GEOS 1 and 2, NASA/ESA ISEE-1, Swedish Viking, Swedish Freja, NASA/USAF CRRES, NASA Polar, FAST, and THEMIS, and ESA/NASA Cluster spacecraft, as well as boom systems on dozens of sounding rockets.

The RBSP axial booms consist of one pair of booms to be deployed along the spacecraft spin axis. As shown in Fig. 12, the booms can be deployed to a maximum length of 7 meters each and are terminated with spherical sensors with internal preamplifiers giving a maximum tip-to-tip separation of $\sim 15$ meters. The boom deployment units are mounted back-to-back within a carbon composite tube installed along the center line between the top and bottom decks of the spacecraft structure. In the stowed configuration, the axial probe is 
held against the spacecraft deck in a caging mechanism. When released, the probe and whip assembly rotate up along the spacecraft spin axis. At that point the whip/probe assembly can be deployed along the spin axis at the end of a stacer boom element. The stacers are spring loaded helical beryllium-copper coils of metal with an outward spring force. Unlike designs for previous spacecraft, the deployed length of this boom is adjustable. The purpose of this capability is to optimize the position of the sensors relative to the asymmetric electrical equipotential associated with the spacecraft. By placing the sensors on the same spacecraft equipotential surface, error offsets to the electric field measurement may be removed. In this design, the outward spring force of the stacer is restrained by the outer Kevlar braid of a cable through the center and along the length of the stacer tube. The in-board end of this cable is wound around a motor driven spool. Powering the motor allows the spool to unwind under the outward spring force of the stacer element. As a result, the deployed boom length can be adjusted with a resolution of $0.5 \mathrm{~cm}$ but only in one direction-there is no retraction capability. The lengths of the booms will be adjusted in stages during the commissioning phase of the mission.

The sensor consists of a $75 \mathrm{~cm}$-long, tapered (4.8 to $7.0-\mathrm{mm})$ graphite-coated (DAG213) whip with $4 \mathrm{~cm}$ radius sphere at its tip. The preamp enclosure is similar to that found on the spin plane booms, and contains the preamp electronics board, as well as acting as two of the photoelectron control surfaces (usher and guard). The preamp and whip assembly is mounted to the outboard end of the graphite coated (DAG-214) main stacer. A two-stage deployment assist device (DAD) is spring-loaded, and serves to start the stacer deploy. The stacer element is formed during deployment through two sets of roller nozzles to provide for lateral stability of the stacer when fully deployed.

As with the spin plane booms, the motor in each unit is shielded with mu metal to limit leakage of stray magnetic fields. The drive mechanism is fully enclosed to provide EMI shielding, as well as to keep debris out. EMI filters are used on both power lines.

\section{Boom Deployment During Commissioning}

Following the successful launch of the RBSP spacecraft on 30 August 2012 and the successful turn-on of the EFW instruments, the spin plane boom deployments began on both spacecraft on September 13, 2012. After opening the exterior doors on the deployment units, the DCB was commanded to deploy each of one pair of Spin Plane Booms to a length of 4 meters corresponding to a total tip-to-tip distance between the two pair (including the spacecraft) of $9.8 \mathrm{~m}$. Following that, the other pair was deployed a similar amount. Over the course of many orbits, this alternating pair-wise deployment continued until all four spin plane booms reached a final deployed length of 50 meters on each boom element giving two orthogonal 100-meter tip-to-tip dipoles. When deployed to a length of about $10 \mathrm{~m}$, the fine-wire between the preamplifier housing and the spherical probe deploys to a length of 3 meters. At several points in the deployment process, the spacecraft spin rate was increased to compensate for the increasing moment-of-inertia such that, when the booms reached their final length on 22 September 2012, the spacecraft spin rate was the nominal 5.48 RPM.

Once the spin plane booms were fully deployed, the axial boom deployments began on both spacecraft on September 24, 2012. After opening the caging mechanisms and confirming proper deployment of the whip/sphere assemblies, the DCB was commanded to deploy one axial boom to a length of $\sim 4$ meters. Following that, the other boom was deployed a similar amount and then the pair on the other spacecraft. After several orbits, the axial boom lengths were adjusted to provide a tip-to-tip sensors separation of $\sim 14$ meters It is expected that further trimming operations will be conducted as needed to optimize the science. 
Analyses of the boom dynamics during deployment, Earth shadow entry and exit and attitude maneuvers for a small spinning spacecraft similar to the baseline RBSP spacecraft indicate that booms pose no stability problems. This analysis is consistent with the flight experience from the previously mentioned spacecraft. On-orbit characteristics of RBSP spin plane boom dynamics are consistent with expectations.

Spacecraft Accommodation of EFW The requirement for a sensitive double probe electric field experiment motivates several important properties of the RBSP spacecraft. The near sun-pointing spin axis is needed so that photo-illumination of the spin plane booms and sensors is uniform over the spin period of the spacecraft.

The necessity for a conducting spacecraft has lead to the coating of the solar cell cover glasses and the thermal blankets with a conductive coating of Indium Tin Oxide (ITO). Conductive paths through wiring of solar panels and grouting between panels allowing current to flow from solar cells to the body of the spacecraft have been implemented. There are strict limits on the total surface area of exterior insulating surfaces and instrument apertures, which could to charge to high potentials. These requirements are designed to give a nearly constant floating potential for the electric field sensors and reduce spin dependent errors in the electric field.

Since the front-side of the spacecraft is illuminated by the Sun and the "back" of the spacecraft is not illuminated, unless otherwise prevented, the front side of the spacecraft will charge to potentials of tens of volts positive while the shadowed portion of the spacecraft will charge to the characteristic thermal energy of the plasma electrons. Experience on previous spacecraft missions and modeling indicate this electric potential can range from $\sim 1$ volt in the high-density low temperature plasmasphere to several kilovolts during intense substorm electron injection events. This would be a major impact on the electric field measurements since it not only contributes large asymmetries ( $100 \mathrm{~V}$ to several $\mathrm{kV})$ to the spacecraft potential structure around the spacecraft but it is sometimes large enough to saturate the electric field pre-amplifiers. The solution to this problem is to insure that both the shadowed and illuminated surfaces on the spacecraft are conductively tied together such that photo-currents on the illuminated surfaces including solar panels are able to flow to the shadowed surfaces where, they dominate, under typical plasma conditions, over currents associated with high energy electrons in controlling current balance and floating potential.

For the same reason, the spin axis boom measurements are accurate only when the antisunward spin axis sensor is not shadowed by the body of the spacecraft or the solar panels. This motivates a pointing strategy in which the spin axis is nominally pointed at least 15 degrees away from the Sun so that the anti-sunward probe and a short section of the adjacent boom element are not shadowed by the spacecraft.

\section{EFW Space Weather Products}

The EFW instrument flight software incorporates a module for the calculation of space weather products for real time telemetry to the ground. EFW space weather products are: (1) spin fit electric field with a time cadence of $\sim 12$ seconds and (2) the spacecraft potential. It should be noted that the electric field data at this level of analysis still includes the motional electric field of the spacecraft $\left(V_{\mathrm{sc}} \times B\right)$ discussed above. These products will provide real time information on the electric fields associated with injection events and interplanetary shock induced waves. The spacecraft potential measurement provides information on thermal structures over the density range from $0.1-50 \mathrm{~cm}^{-3}$ for cold plasma regimes 
$(<10 \mathrm{eV})$. The spacecraft potential is an important diagnostic for the detecting discontinuities in thermal plasma including the plasmapause and the tail-lobe plasma sheet boundary, and, occasionally, the magnetopause. Calibration files for these products may be found on the EFW SOC website. This dataset is designed for real time and near real time diagnostics of the space weather of the inner magnetosphere and not for publication purposes.

Data Collection, Analysis, and Dissemination and Archiving The EFW Science Operations Center (SOC) is located at SSL/UCB. Instrument command loads and mode schedules are generated and verified at the EFW SOC based on the overall RBSP mission plan, the EFW science plan, and other input from the EFW science team. These command loads are sent to the Mission Operations Center (MOC) operated by APL. In addition to its role in monitoring and maintaining instrument health and safety and in instrument commanding, the SOC will provide access to the EFW data and software products. The EFW SOC downloads telemetry data from the RBSP MOC; provides secure and redundant archiving of the all data; produces and validates the EFW Level 1 CDF data products; produces and validate the EFW Level 2 CDF data products; and, makes these data products widely available.

SSL has developed and implemented an automated system for level 0 data acquisition from NASA sites that is currently used to electronically download the THEMIS, STEREO, Wind, and RBSP EFW data sets. The SOC at the SSL electronically downloads the level 0 data and the additional spacecraft housekeeping data and ancillary data products from the RBSP MOC, and produces time tagged level 1 data, and also provides the calibrated science quantities comprising the level 2 processed data in CDF files. The EFW level 2 CDF files are available on-line. The SOC records and archives the EFW data, and produces daily quicklook science survey plots. The survey plots are placed on line for access by the scientific community. The summary plots are designed as a "first step" browse tool to identify events. The summary data is not a final scientific product. It contains known errors associated with incorrect or in-appropriate biasing, episodes of preamplifier saturation associated spacecraft charging, spin axis sensor shadowing by solar panels, and other spurious signals. This data is not to be published in the scientific literature. Researchers are encouraged contact the P-I when using any EFW data for presentations and publications.

Scientists interested in the complete EFW data set can download the analysis software and data set from the SOC website. The SOC web site will contain a complete set of help pages that detail the format and data quantities available in the CDF files, the data available in the complete data set and provide assistance in installation and use of the analysis software. Software analysis tools include IDL based TPLOT, and TDAS software routines first developed at SSL for FAST and THEMIS particle and fields measurements. In addition, the SDT analysis program used in Polar, Cluster and THEMIS data analysis is available. These analysis tools allow electric field data to be rotated into a variety of geophysical coordinates, calculation of spin fit electric field vectors, subtraction of the spacecraft motional electric field, generation of spectra products, as well as, more detailed scientific analysis.

\section{Conclusion}

Since early commissioning and checkout, measurements from EFW instruments on the two RBSP spacecraft, in concert with the other fields and particle instruments on the spacecraft, have been providing unprecedented capabilities to investigate the physics of energetic particle acceleration and the dynamics of major geomagnetic storms in the inner magnetosphere. The unique insights provided by these measurements should allow a conceptual framework for a central problem in space and astrophysics which is based on direct in-situ observations of the structures and waves controlling the acceleration of energetic particles. 
Acknowledgements We would like to acknowledge the many contributions of the RBSP Project team at Johns Hopkins Applied Physics Laboratory. We would like to also thank the EFW team of scientists and engineers at Space Science Laboratory at the University of California, Berkeley and the staff at the Laboratory for Atmospheric and Space Physics at the University of Colorado in the development and test of the instrument. We also thank the efforts of the other instrument teams on the RBSP mission. We would like to acknowledge the contributions of the EMFISIS team, the ECT team (MagEIS, HOPE and REPT), and the RBSPICE team. In this paper, the energetic electron figures were provided by Joe Fennell, J.B. Blake, and Harlan Spence of the MagEIS/ECT teams. This work was supported by RBSP-ECT funding provided by JHU/APL Contract No. 967399 under NASA's Prime Contract No. NAS5-01072. The work by the EFW team was conducted under JHU/APL contract 922613 (RBSP-EFW).

Open Access This article is distributed under the terms of the Creative Commons Attribution License which permits any use, distribution, and reproduction in any medium, provided the original author(s) and the source are credited.

\section{References}

J.M. Albert, Gyroresonant interactions of radiation belt particles with a monochromatic electromagnetic wave. J. Geophys. Res. 105(A9), 21 (2000). 191

W.I. Axford, Flow of mass and energy in the Solar system, physics of solar planetary environments, in Proceeding of the International Symposium on Solar-Terrestrial Physics, vol. 1 June 7-18 American Geophysical Union (1976), pp. 270-283

J.B. Blake, W.A. Kolasinski, R.W. Fillius, E.G. Mullen, Injection of electrons and protons with energies of tens of MeV into $L<3$ on 24 March 1991. Geophys. Res. Lett. 19, 821 (1992)

J.B. Blake, S.S. Imamoto, The proton switches. J. Spacecr. Rockets 29, 595 (1992)

J.B. Blake, M.D. Looper, D.N. Baker, R. Nakamura, B. Klecker, D. Hovestadt, New high temporal and spatial resolution measurements by SAMPEX of the precipitation of relativistic electrons. Adv. Space Res. 18(8), 171-186 (1996)

J.B. Blake et al., The ECT instrument on the Van Allen probes (this issue)

J.W. Bonnell et al., The electric field instrument for THEMIS. Space Sci. Rev. 114, 303-341 (2008). doi:10.1007/s11214-008-9469-2

D.H. Brautigam, G.P. Ginet, J.M. Albert, J.R. Wygant, D.E. Rowland, A. Ling, J. Bass, CRRES electric field power spectra and radial diffusion coefficients. J. Geophys. Res. 110, A02214 (2005). doi:10.1029/2004JA010612

C. Cattell et al., Polar observations of solitary waves at low and high altitudes and comparison to theory. Adv. Space Res. 28, 1631 (2001)

C. Cattell et al., Discovery of very large amplitude whistler-mode waves in Earth's radiation belts. Geophys. Res. Lett. 35, L01105 (2008). doi:10.1029/2007GL032009

C.M. Cully, J.W. Bonnell, R.E. Ergun, THEMIS observations of large amplitude whistler mode waves in the inner magnetosphere. J. of Geophys. Res. 35, L17S16 (2008). doi:10.1029/2008GL033643

L. Dai et al., Observations of surface waves in jets from magnetotail reconnection. J. Geophys. Res. 116, A12227 (2011). doi:10.1029/2011JA017004

J. Dombeck et al., Observed trends in auroral zone ion mode solitary structure characteristics using data from polar. J. Geophys. Res. 106, 19013 (2001)

R.E. Ergun et al., The FAST satellite field instrument. Space Sci. Rev. 98, 67-91 (2001)

G. Gustafsson et al., The electric field and wave experiment for the CLUSTER mission. Space Sci. Rev. 79, 137 (1997)

P. Harvey, F.S. Mozer, D. Pankow, J. Wygant, N.C. Maynard, H. Singer, W. Sullivan, P.B. Anderson, A. Pedersen, C.-G. Falthammar, P. Tanskannen, in The Electric Field Instrument on the Polar Satellite in the Global Geospace Mission, vol. 71, ed. by C.T. Russell (Kluwer Academic, Dordrecht, 1995). Reprinted from Space Sciences Rev., 71, N1-4, 1995

R.B. Horne, R.M. Thorne, Potential waves for relativistic electron scattering and stochastic acceleration during magnetic storms. Geophys. Res. Lett. 25(15), 3011-3014 (1998)

M.K. Hudson, A.D. Kotelnikov, X. Li, I. Roth, M. Temerin, J. Wygant, Simulation of proton radiation belt formation during the March 24, 1991 SSC. Geophys. Res. Lett. 22, 291 (1995)

M.K. Hudson, S.R. Elkington, J.G. Lyon, V.A. Marchenko, I. Roth, M. Temerin, J.B. Blake, M.S. Gussenhoven, J.R. Wygant, Simulations of radiation belt formation during storm sudden commencement. J. Geophys. Res. 102, 14087 (1997) 
M.K. Hudson, S.R. Elkington, J.G. Lyon, M.J. Wiltberger, in Radiation Belt Electron Acceleration by ULF Wave Drift Resonance: Simulation of 1997 and 1998 Storms, ed. by G. Siscoe, P. Song, H. Singer. AGU Monograph, Space Weather (AGU, Washington, 2001), p. 289

M.K. Hudson, R.E. Denton, M.R. Lessard, E.G. Miftakhova, R.E. Anderson, A study of Pc5 ULF oscillations. Ann. Geophys. 22, 289 (2004)

J.C. Ingraham, T.E. Cayton, R.D. Belian, R.A. Christensen, R.H.W. Friedel, M.M. Meier, G.D. Reeves, M. Tuszewski, Substorm injection of relativistic electrons to geosynchronous orbit during the great magnetic storm of March 24, 1991. J. Geophys. Res. 106(A11), 25759-25776 (2001). doi:10.1029/2000JA000458

P.J. Kellogg et al., Electron trapping and charge transport by large amplitude whistlers. Geophys. Res. Lett. 37, L20106 (2010). doi:10.1029/2010GL044845

K. Kersten et al., Observation of relativistic electron microbursts in conjunction with enhanced radiation belt whistler mode waves. Geophys. Res. Lett. 38, L08107 (2011). doi:10.1029/2001GL046810

K. Kirby et al., The radiation belt storm probes-observatory and environment (this issue)

C. Kletzing et al., The electric and magnetic field instrument suite and integrated science and integrated science (EMFISIS) on RBSP. Space Sci. Rev. (2013). doi:10.1007/s11214-013-9993-6 (this issue)

X. Li, I. Roth, M. Temerin, J.R. Wygant, M.K. Hudson, J.B. Blake, Simulation of prompt energization and transport of radiation belt particles during the March 24, 1991 SSC. Geophys. Res. Lett. 20, 2423 (1993)

X. Li, D.N. Baker, M. Temerin, G. Reeves, R. Belian, Simulation of dispersionless injections and drift echoes of energetic electrons associated with substorms. Geophys. Res. Lett. 25, 3763 (1998)

K.R. Lorentzen, J.E. Mazur, M.D. Looper, J.F. Fennell, J.B. Blake, Multisatellite observations of MeV ion injections during storms. J. Geophys. Res. 107, 1231 (2002)

G. Marklund, Viking investigations of auroral electrodynamical processes. J. Geophys. Res. 98, 1691 (1993)

G. Marklund et al., The Swedish small satellite program for space plasma investigations. Space Sci. Rev. 111(3-4), 377-413 (2004). doi:10.1023/B:SPAC.0000032690.82775.dB

R.M. Millan, R.P. Lin, D.M. Smith, K.R. Lorentzen, M.P. McCarthy, X-ray observations of MeV electron precipitation with a balloon-borne germanium spectrometer. Geophys. Res. Lett. 29, 2194 (2002)

R.M. Millan (this issue)

F. Mozer, Analysis of techniques for measuring DC and AC electric fields in the magnetosphere. Space Sci. Rev. 14, 272 (1973)

F.S. Mozer et al., A proposal to measure quasi-static electric fields on the ISEE-1 mother daughter satellite, University of California Berkeley Space Science Technical Note, UCBSSL No. 454 (1973)

F.S. Mozer et al., The dc and ac electric field, plasma density, plasma temperature and field-aligned current experiments on the S3-\# spacecraft. J. Geophys. Res. 84(A10), 5875 (1979)

T.P. O'Brien, K.R. Lorentzen, I.R. Mann, N.P. Meredith, J.B. Blake, J.F. Fennell, M.D. Looper, D.K. Milling, R.R. Anderson, Energization of relativistic electrons in the presence of ULF power and MeV microbursts: evidence for dual ULF and VLF acceleration. J. Geophys. Res. 108(A8), 1329 (2003). doi:10.1029/2002JA009784

A. Pedersen, Solar wind and magnetospheric plasma diagnostics by spacecraft electrostatic potential measurements. Ann. Geophys. 13, 118 (1995)

A. Pedersen et al., Electron density estimations derived from spacecraft potential measurements on cluster in tenuous plasma regimes. J. Geophys. Res. 113, A07S33 (2008). doi:10.1029/2007JA012636

G. Reeves et al. (this issue)

I. Roth, M. Temerin, M.K. Hudson, Resonant enhancement of relativistic electron fluxes during geomagnetically active periods. Ann. Geophys. 17, 631 (1999)

D. Rowland, J.R. Wygant, The dependence of the large scale electric field in the inner magnetosphere on magnetic activity. J. Geophys. Res. 103(A7), 14959 (1998)

D. Rowland, The electrodynamics of the inner magnetosphere during major geomagnetic storms. PhD Thesis, University of Minnesota (2002)

O. Santolik, D.A. Gurnett, J.S. Pickett, M. Parrot, N. Cornilleau-Wehrlin, Spatio-temporal structure of stormtime chorus. J. Geophys. Res. 108, 1278 (2003). doi:10.1029/20002JA009791

H. Spence et al. (this issue)

D. Summers, Y. Omura, Ultra-relativistic acceleration of electrons in planetary magnetospheres. Geophys. Res. Lett. 34, L24205 (2007). doi:10.1029/2007GL032226

A. Vampola, H. Korth, Electron drift echoes in the inner magnetosphere. Geophys. Res. Lett. 19, 625 (1992)

L. Wilson et al., The properties of large amplitude whistler mode waves in the magnetosphere: propagation and relationship with geomagnetic activity. Geophys. Res. Lett. 38, L17107 (2011). doi:10.1029/2011GL048671

J.R. Wygant, P.R. Harvey, D. Pankow, F.S. Mozer, N. Maynard, H. Singer, M. Smiddy, W. Sullivan, P. Anderson, The CRRES electric field experiment/Langmuir probe. J. Spacecr. Rockets 29, 601 (1992) 
J.R. Wygant, F. Mozer, M. Temerin, J. Blake, N. Maynard, H. Singer, M. Smiddy, Large amplitude electric and magnetic field signatures in the inner magnetosphere during injection of $15 \mathrm{MeV}$ drift echoes. Geophys. Res. Lett. 21, 1730 (1994)

J.R. Wygant, H.J. Singer, M. Temerin, F. Mozer, M.K. Hudson, Experimental evidence on the role of the large spatial scale electric field in creating the ring current. J. Geophys. Res. 98, JA01436 (1998)

H.-C. Yeh, J.C. Foster, F.J. Rich, W. Swider, Storm-time electric field penetration observed at mid-latitude. J. Geophys. Res. 96, 5707-5721 (1991) 